\title{
Sono condivisibili e implementabili nel mondo reale i percorsi suggeriti? Quali sono i veri ostacoli allo sviluppo della riabilitazione-prevenzione e come superarli?
}

\author{
Monaldi Arch Chest Dis 2006; 66: 147-160.
}

\section{F. CHIARELLA (ANMCO)}

In era interventistica, se noi pensiamo ad una sceneggiatura in atto negli ospedali italiani, probabilmente in questo momento un paziente sta uscendo da una sala di emodinamica e l'emodinamista gli dirà "abbiamo aperto la coronaria, può andare". Ci sarà un primario che, insieme con il suo collaboratore, sta visitando i pazienti e chiederà: "Questo? tre giorni. Bene, domani lo dimetti”. Dall'altra parte c'è un altro scenario, quello del medico di medicina generale che sta ricevendo un paziente dimesso da una settimana visibilmente in ansia, agitato che ha avuto una precordialgìa, e a cui sta dicendo: "Beh, a questo punto vai al pronto soccorso". Questo per dire la realtà, e quanto siamo lontani dalle cose che abbiamo sentito.

Vorrei elencare tre tipi di problema e tre tipi di ostacoli.

Il primo problema che vedo in termini concreti è il paziente, di cui bisogna prendersi cura completamente. $\mathrm{Ci}$ sono molte cose che si possono fare isorisorse, ma che non si fanno, ci sono delle cose che invece richiedono sicuramente immissione di risorse e dobbiamo vedere e individuare quali queste possono essere.

Il secondo problema è la cultura. Nella nostra realtà di cardiologia italiana siamo ancora lontani da una cultura che preveda l'individuazione dei fattori di rischio come un vero bersaglio, e da una educazione alle persone che stanno svolgendo il loro lavoro, per dare a questi la dignità che meritano. Noi sappiamo quanto pesa in Italia la cardiopatia ischemica ed in particolare le malattie cardiovascolari, l'arteriosclerosi, sappiamo a che punto è nella scala epidemiologica, ma non ci rendiamo ancora conto dell'importanza che senza impiego di nuove risorse venga individuato sistematicamente un tempo, un soggetto che spieghi al paziente che cosa sono i fattori di rischio, cosa di cui andiamo dicendo da tanto tempo, e cosa altrettanto negata nella realtà quotidiana. Quindi c'è un problema di cultura, ma si tratta di una cultura che deve essere interdisciplinare, e tutta protesa verso una continuità assistenziale. Sta di fat- to che questo ponte stenta ad attivarsi e che per attivare questo ponte ci vogliono obiettivi precisi, motivazioni forti da parte delle persone, ma innanzitutto ci vuole la ripresa dell'attenzione verso il paziente non come un pezzo di qualche cosa, ma come un'unità intera da seguire. Credo che, se si riuscisse a riflettere maggiormente sulla unitarietà del paziente come persona, molti passaggi sarebbero più facili.

Il terzo problema è sicuramente quello delle risorse. Ora è vero che negli ospedali molto spesso gli organici sono insufficienti. È vero che il tempo manca sempre di più, è vero che non si può continuare con così poche risorse, però è anche vero, e mi consta, che siano state allocate delle risorse sulla prevenzione verso le regioni, e le regioni le hanno allocate verso qualche cosa che non è ancora, almeno in molte realtà, definito. Spesso queste risorse sono state indirizzate verso commissioni, non meglio precisate, che dovrebbero contenere al loro interno tante figure, e i cardiologi sono la 'cenerentola' di queste figure. Se dobbiamo fare un discorso complessivo, dobbiamo anche pensare che allora tutte le risorse della prevenzione sono allocate sui medici di medicina generale, e questo significa in qualche modo allargare ancor di più il solco che forse c'è. Si tratta di ripensare ad un'operazione complessiva e non soltanto settoriale, si tratta di coinvolgere un soggetto che sta dentro l'ospedale e fuori dall'ospedale, quindi si tratta effettivamente di fare un'azione intelligente perché le regioni che hanno ricevuto risorse in questo senso le utilizzino al meglio, non parzialmente o per finalità che non sono consone ad un vero programma di prevenzione che potrebbe anche prevedere un supporto ad alcuni passaggi o ad alcune figure, penso ai fisioterapisti, o ad alcuni momenti che vedono tanto l'ospedale, quanto la fase post-ricovero, post-degenza. Questo in sintesi quello che posso dire come ANMCO.

\section{G. GREGORIO (ANMCO)}

Mi ricollego a quanto detto da Franco Chiarella. Fondamentalmente i problemi sono di due tipi: vi è 
un problema culturale, legato all'habitus che ha il cardiologo oggi, che sta perdendo sempre di più le sue radici legate ad una disciplina cardiologica nel senso completo della parola. Viviamo in un'epoca in cui si sente sempre più il bisogno di dire: "Io sono emodinamista, io sono elettrofisiologo, io sono ecocardiografista", e questo fatto non è semplicemente dovuto all'ipertecnologia, è dovuto ad un radicale cambiamento della mentalità con cui si lavora nei reparti di cardiologia. E sostanzialmente è sempre più evidente che l'emodinamista finisce per non sapere nulla dell'ammalato che ha trattato in acuto, che viene affidato poi ad un altro medico in reparto e spesso in altri reparti, e se non c'è questa prima fase di colloquio all'interno di questa struttura, penso che sia molto difficile ritenere che vi possa essere un discorso aldilà delle mura dell'ospedale. Fondamentalmente noi viviamo in un mondo sanitario che continua ad essere rigidamente, aldilà delle enunciazioni anche legislative, un sistema a compartimenti stagni. La regione Campania ha probabilmente prodotto le migliori leggi che vi sono in Italia; abbiamo scritto il percorso dell'urgenza-emergenza in una legge fatta nel '94 (la prima legge organica sul sistema dell'emergenza), abbiamo dovuto aspettare 10 anni dal '94 perché in questa regione forse poi attuato il 118. Abbiamo un'altra legge del '98 che fissa i percorsi cardiologici e che sostanzialmente recepisce il documento dell'ANMCO sui modelli organizzativi ma che per buona parte, nonostante fosse un piano ospedaliero che aveva la forza di legge, è rimasta sulla carta. Questa è la storia di tutte le leggi di questo paese. Quindi vi è un primo problema di cultura, che riguarda il cardiologo ospedaliero e che si riflette poi nella organizzazione in generale. Siamo un mondo che rimane diviso, aldilà delle buone intenzioni della Federazione. Esiste un'associazione di cardiologi ospedalieri, un' associazione di cardiologi extra-ospedalieri, continuano ad esistere una serie di associazioni di settore con proprie logiche e propri comportamenti. Oltre a questo problema di cultura, vi è un problema organizzativo. Dobbiamo tendere ad un modello organizzativo che superi il concetto delle mura dell'ospedale. Personalmente dirigo, in una delle aziende sanitarie della Campania più vasta, all'incirca la metà della provincia di Salerno, sono $3000 \mathrm{Kmq}$, un dipartimento ospedaliero di medicina cardiovascolare che raggruppa sei divisioni di cardiologia. È un dipartimento solo ospedaliero, non abbiamo praticamente grandi colloqui con l'assistenza primaria, i cosiddetti medici di medicina generale, il $99 \%$ dei pazienti che giunge in ospedale vi giunge nella maggior parte dei casi spontaneamente, o per lo meno senza la relazione del medico di assistenza primaria, esiste poco colloquio con la specialistica ambulatoriale, che abbiamo tentato di superare condividendo dei percorsi. Non è così semplice mettere in pratica un percorso assistenziale. Come diceva Carlo Schweiger, viviamo in un'epoca in cui fondamentalmente ad ognuno di noi arrivano i reports delle nostre attività che sono una serie di prestazioni erogate. La prestazione è slegata dal paziente ed è difficile ricondurre l'insieme di prestazioni ad una logica di percorso, e ad una logica di pazienti e non credo che onestamente sia un percorso molto semplice e molto facile da attuare.

\section{A. BALBARINI (SIC)}

Ho identificato fondamentalmente tre punti che le due società e la federazione dovrebbero portare avanti per promuovere e migliorare gli aspetti della riabilitazione. Il primo punto, secondo me, riguarda l'informazione: trasferire cultura alla popolazione. Anche il paziente che ha un evento cardiovascolare dovrebbe capire l'importanza della riabilitazione, della prevenzione, che sono poi strettamente collegate. A questo proposito ho notato, ma è anche una realtà toscana, che la riabilitazione del paziente post-chirurgico si fa in quantità elevata, ma si fa molto meno la riabilitazione nell'infartuato. Da dati che mi ha forniti dal dott. Giustarini, responsabile del centro Auxilium Vitae di Volterra, mi risulta che in Toscana dovrebbero essere riabilitati circa 8000 infartuati che non lo sono. Quindi questo secondo me è un problema importante che le due società e la federazione dovrebbero portare avanti, cioè informazione, trasferimento della cultura alla popolazione. Il secondo punto ugualmente importante: informare, ma soprattutto convincere, chi ci deve dispensare di risorse. Oggi è difficile avere risorse in questo settore, cioè è chiaro che le risorse vengono trasferite nelle situazioni acute cardiovascolari e questo è comprensibile quando abbiamo le carenze di risorse che la sanità lamenta. Ma noi dovremmo far capire a chi ci governa, che investire oggi in riabilitazione, in prevenzione, vuol dire risparmiare domani, e quindi questo è un messaggio che dobbiamo trasferire fortemente. Il terzo punto è stato illustrato in maniera ampia ed esauriente dal Prof. Gensini e però mi piace ribadire alcuni concetti. $\mathrm{Ci}$ sono in effetti le cosiddette lauree brevi che preparano i riabilitatori, ma sono rivolte ovviamente a personale non medico, come c'è Scienze Motorie. Trovo una carenza di preparazione e di formazione, soprattutto per i medici. La prima carenza riguarda anche la preparazione degli studenti di Medicina, non ci sono sufficienti messaggi formativi ai medici. Nella mia esperienza, se chiedete ad uno studente di Medicina di riferire quelli che sono gli effetti per esempio dell'esercizio fisico sull'apparato cardiovascolare, le nozioni sono assolutamente insufficienti. Il fisiologo non lo fa, noi lo facciamo ma naturalmente in maniera non completa, per esempio quando parliamo dei fattori di rischio, invece questo secondo me è un settore in cui la formazione dovrebbe essere aumentata. Vi ricordo le scuole di specializzazione di cui ha parlato il prof. Gensini. C'è una scuola di medicina fisica riabilitativa, che però prevalentemente, è rivolta alla riabilitazione ortopedica e neurologica; poco si fa per quanto riguarda la riabilitazione cardiologica. Per fortuna, il Legislatore, come avete visto, ha sopperito questa carenza e nella nuova scuola di Cardiologia che si chiamerà "di Malattia dell'apparato cardiovascolare", c'è un riferimento preciso a quella che è la riabilitazione. Bene, però il messaggio del Prof. Gensini è quello che ciascuno di noi dovrebbe prendere delle iniziative già adesso perché la scuola se non sbaglio dovrebbe entrare in vigore nel 2007. Per esempio, a Pisa abbiamo preso un'iniziativa in questo senso. La scuola di Cardiologia ha una convenzione con l'Auxilium Vitae, uno specializzando di cardiologia passa un periodo a rotazione nella 
struttura riabilitativa, ed è stato chiesto anche un bando di concorso per un professore a contratto in questo settore. Quindi è importante anche che le iniziative personali, locali ed altro vadano in questa direzione. Quindi i tre punti che secondo me le società dovrebbero portare avanti sono quelli che vi ho detto e dobbiamo ben tener presente che soltanto con una prevenzione adeguata noi potremmo diminuire le complicazioni della cardiopatia ischemica, che è la prima malattia che interessa l'apparato cardiovascolare.

\section{A. CAMPANA (AIAC)}

È concettualmente importante che l'AIAC sia presente a questa Tavola Rotonda. Il 10 Febbraio sono state pubblicate su Internet le linee guida nazionali su "Cardiologia riabilitativa e prevenzione secondaria delle malattie cardiovascolari", a cura della Agenzia per i Servizi Sanitari Regionali.

Su 265 voci bibliografiche trovate ai margini di queste linee guida, ce ne sono soltanto due che riguardano in qualche modo la riabilitazione in pazienti aritmici: un'editoriale pubblicato su Heart nel 2001, riguardante la opportunità di sottoporre pazienti con impianto di defibrillatore automatico ad una terapia globale di riabilitazione psicofisica, e uno studio scozzese, molto limitato, pubblicato sempre su Heart nel 2003, in cui si sottoponevano 16 pazienti ad un ciclo riabilitativo di dodici settimane, rilevando nei pazienti un certo miglioramento nella capacità di eseguire esercizi fisici, e un notevole miglioramento dell'indice di depressione e di ansietà, misurato con una scala apposita (ADH). Nei pazienti portatori di defibrillatore, una prima impressione di maggiore sicurezza perché protetti dalla morte improvvisa, viene seguita poi dalla insorgenza di depressione nelle settimane successive. Credo, per quanto riguarda questo aspetto, che i portatori di defibrillatore siano pazienti che già per altri motivi, perché ischemici o scompensati, sarebbero dovuti rientrare in programmi di riabilitazione; essi sicuramente costituiscono un sottogruppo particolare, perché chi ha fatto tentativi di sottoporre a sforzo controllato elettrocardiograficamente i pazienti aritmici, ha visto come la percentuale di insorgenza di aritmie importanti nei pazienti "aritmici" è sensibilmente superiore rispetto ad un gruppo di pazienti di confronto. Credo che questo sia un problema da porsi; sicuramente l'AIAC dovrà affrontarlo, studiando il modo in cui si possa dare un supporto continuo anche psicologico a questa categoria di pazienti, soprattutto portatori di defibrillatore e di pacemaker. Certamente noi aritmologi siamo avvantaggiati in questo, potendo utilizzare, espandendole, le capacità e le funzioni degli specifici ambulatori di controllo di cui disponiamo. Voi tutti sapete che i portatori di defibrillatori e pacemaker sono seguiti periodicamente nei Centri d'impianto; esistono degli ambulatori sparsi su tutto il territorio nazionale dove i portatori di "device" vengono chiamati a controllo con appuntamenti regolari: questa opportunità potrebbe essere implementata con programmi di supporto psicologico, anche in sedute di gruppo.

\section{G. ZITO (ARCA)}

Io rappresento la cardiologia ambulatoriale, vale a dire quella parte della cardiologia che potrebbe rivestire un importante ruolo di cerniera tra tutte queste componenti in questa fase in cui è presente un po' di scollamento, di solitudine, come diceva Klugmann nel suo intervento. Ritengo che questa attività può avere un ruolo molto importante, anche all'interno delle cose che abbiamo detto e delle prospettive di organizzazione che abbiamo già citato. Due elementi forti vengono fuori: prima di tutto la mancanza di una cultura comune tra le varie componenti della cardiologia: cardiologia del territorio, cardiologia ospedaliera e cardiologia universitaria. È questo un dialogo difficile, perché bisogna cambiare una mentalità che appartiene in misura diversa ad ognuno di noi. Un secondo elemento è quello di partire dall'esistente, andando per un attimo aldilà delle possibilità future che si possono disegnare, alcune particolarmente interessanti e particolarmente utili per i pazienti se si riuscissero a realizzare. Ripeto, alcune ipotesi e prospettive future sono particolarmente interessanti, ma io credo che l'esistente già possa rappresentare qualcosa che sia capace di creare un meccanismo nuovo, che possa agevolare il dialogo tra le varie componenti della cardiologia. Consentitemi un breve inciso: la Federazione Italiana di Cardiologia, grazie alla ferrea volontà di Gianluigi Nicolosi, ha pubblicato qualche tempo fa una monografia e nell'ambito di questa ho avuto il piacere e l'onore di avere l'incarico di disegnare un'ipotesi di collegamento tra ospedale e territorio, nella gestione del paziente cardiopatico. La mia ipotesi partiva proprio da una fotografia di quello che oggi c'è per poi allontanarsi su proposte che, necessitando di modifiche complesse da un punto di vista organizzativo, possono essere particolarmente utili ma non in tempi brevi. Io ritengo che una continuità assistenziale ospedale-territorio debba muoversi su tre livelli, che non vanno vissuti come dei compartimenti stagni, ma con possibilità di interscambio costante. Un primo livello deve essere rappresentato dai medici che svolgono il lavoro elettivamente sul territorio. Vale a dire i colleghi di medicina generale, che nella gestione del paziente cardiopatico possono essere supportati per le necessità che si possono creare dai cardiologi ambulatoriali. Cioè si deve creare un livello territoriale puro dove il paziente può essere gestito nelle fasi di stabilizzazione e dove è possibile attivare tutte le procedure di cura e prevenzione per evitare ospedalizzazioni e/o riospedalizzazioni. Un secondo livello può essere rappresentato dai cardiologi ospedalieri, a cui il nucleo territoriale può far ricorso, oltre nelle fasi acute di instabilizzazione, anche per indagini strumentali più complesse che non possono essere svolte a livello territoriale. Un terzo livello deve essere rappresentato dai centri di eccellenza (ospedalieri ed universitari), strutture indispensabili per i casi più complessi. Questo terzo livello potrebbero coordinare, secondo la strategia dell'Hub and Spoke, le attività degli altri livelli. Se facciamo mente locale, queste sono cose che già abbiamo. C'è una fitta rete territoriale rappresentata dalla medicina generale, esiste in Italia (unica realtà al mondo) una efficace rete di cardio- 
logi ambulatoriali, esistono gli ospedali intermedi e, ovviamente, le strutture di eccellenza. Il vero problema è che non ci si parla. Personalmente ho un ottimo rapporto con $\mathrm{i}$ colleghi universitari, con gli ospedalieri e con i medici di medicina generale della zona dove lavoro, ma il discorso non può essere legato ai rapporti del singolo, si dovrebbe istituzionalizzare il concetto che il paziente è un bene comune e che il nostro obiettivo fondamentale è quello di salvaguardare la sua salute. Ciò non può avvenire se non riusciamo a dialogare tra di noi. Oggi abbiamo l'opportunità di poter finalmente muovere le acque se partissimo da questa ipotesi e se da subito, riuscissimo a gestire insieme in modo coerente, in accordo con le linee guida e con gli obiettivi terapeutici che la moderna cardiologia deve avere spostandoci poi, in un secondo momento, su proposte più complesse.

\section{F. FATTIROLLI (GICR)}

L'auspicio è che da questa conferenza possa derivare, oltre al piacere di essere stati insieme e di aver sentito molti contributi di sicuro interesse, una crescita non solo nelle nostre convinzioni, ma anche nella nostra capacità di rendere operative una serie di cose che ci siamo detti. È un passaggio difficile, ma che può dare il senso allo sforzo che ognuno ha posto in quest'iniziativa. Credo che un obiettivo debba essere quello di porre alcune basi per stilare dei documenti condivisi. Vorrei identificare due problemi: il primo è rappresentato dalla necessità di una migliore messa a punto dell'offerta della cardiologia riabilitativa. Quello che talora nella offerta della cardiologia riabilitativa manca è la flessibilità; spesso da fuori viene visto un modello abbastanza rigido al quale bisogna aderire assolutamente o con il quale è molto difficile interagire. In realtà, invece, le potenzialità culturali, operative e l'esperienza che ormai deriva da molti anni di pratica della cardiologia riabilitativa fanno si che questa flessibilità, che esiste, debba essere meglio esplicitata. Inviterei i partecipanti a questa tavola a dare la loro disponibilità a condividere alcuni percorsi comuni sui quali c'è consenso. Parliamo non solo del paziente che ha meno difficoltà di entrare in questo percorso, per esempio il paziente post-chirurgico, ma del paziente ischemico post sindrome coronarica acuta, che è quello che ha più difficoltà ad entrare nel percorso: Se diciamo che tutti i pazienti dovrebbero accedere alla riabilitazione cardiologica, come dicono le raccomandazioni, sappiamo che questo non è realistico, non esiste nemmeno una capacità di offerta in questo senso. Pertanto dovremmo definire con chiarezza, e condividere con gli emodinamisti e con gli altri colleghi, la scelta di quali percorsi per quali pazienti. Per quali pazienti dovrebbe essere obbligatorio l'accesso alla riabilitazione cardiologica? quali sono i pazienti per i quali invece si possono trovare modelli assistenziali differenziati? Abbiamo un'offerta che alcune volte non viene chiaramente identificata: vi sono Centri di riferimento (centri “hub", definiti così nel documento $\mathrm{ANMCO}$ ) che ricevono pazienti post-chirurgici, pazienti scompensati ancora in fase di instabilità, pazienti con comorbilità o pazienti ad alto rischio. Ma poi ci sono i pazienti con sindrome coronarica non complicata, ma che hanno alta concentrazione di fattori di rischio. Per questi pazienti sono necessari percorsi diversi che si svolgano anche in strutture ed in situazioni organizzative diverse. La nostra capacità deve essere quella di trovare i punti di accordo e di formalizzare i percorsi di base. La flessibilità è presente in tutte le situazioni in cui si ha maggior cultura, maggior conoscenza, e questo si ottiene con un costante impegno formativo, di educazione e aggiornamento continuo su questi temi. La mia proposta è di passare da un utilizzo non codificato del rapporto fra la cardiologia per acuti quella del post-acuto e del cronico, che è alcune volte opportunistico (ad esempio: "questo paziente me lo prendi a fare un po' di riabilitazione?"), ad un rapporto tipo: "questo paziente rientra in quelle caratteristiche che abbiamo detto, per cui deve essere seguito obbligatoriamente in riabilitazione". Poi ci saranno, anche secondo le diverse condizioni organizzative delle strutture, delle differenze locali e geografiche, la possibilità di estendere anche l'offerta, o di differenziarla con un rapporto molto più organico, in cui la potenzialità dell'offerta data dall'esperienza della struttura riabilitativa può essere unita a quella della cardiologia del territorio e del medico di medicina generale.

\section{R. GRIFFO (GICR)}

È stato molto importante che in questa Conferenza parlassero soprattutto gli altri e che noi che ci occupiamo full time di riabilitazione stessimo un po' ad ascoltare. A mio parere sono venute fuori alcune criticità.

1) Gli ostacoli e le barriere alla riabilitazione ben noti a noi cardiologi riabilitatori (li abbiamo indicati anche sulle linee guida dell'Agenzia Regionale) mi sembrano condivisi da parte di quelli che non si occupano a tempo pieno di cardiologia riabilitativa, e credo che la consapevolezza delle barriere e degli ostacoli sia un elemento fondamentale per poterli poi superare.

2) La formazione non solo del del laureando in medicina e dello specializzando, ma anche del cardiologo cosiddetto maturo, cioè quello che c'è qui oggi, e che frequenta generalmente i congressi. Credo che, oltre all'Università anche le società scientifiche generaliste (SIC e ANMCO) abbiano una grande responsabilità. Se credono realmente, così come ripetutamente ribadiscono, che l'intervento riabilitativo e la prevenzione secondaria siano un elemento cruciale del percorso assistenziale dei pazienti cardiopatici, devono inserire in maniera sistematica nei simposi di maggior rilievo nei più importanti congressi generalisti anche l'intervento riabilitativo, fornendo ai partecipanti la presentazione di un percorso assistenziale completo nelle principali patologie cardiovascolari. Questo servirebbe anche per evitare che nei grandi congressi nazionali la riabilitazione venga ghettizzata in simposi dedicati agli specialisti che si parlano tra di loro come accade anche tra gli gli altri superspecialisti. Credo che bisognerebbe affrontare questo problema della formazione permanente del cardiologo offrendo delle op- 
portunità di aggiornamento in cui non venga presentata in modo frammentario la realtà assistenziale del nostro paziente. Il percorso, più volte richiamato oggi, dovrebbe essere identificabile anche dalla costruzione di un simposio.

3) L'altra criticità è anche però legata all'attuale strutturazione del nostro Sistema Sanitario. Qui mi distacco dal consiglio dei moderatori di guardare le cose in un'ottica solo di settore, ma tento di contestualizzare il problema in un ambito generale. Faccio una piccola constatazione: si parla tanto di continuità assistenziale solo da quando gli ospedali sono stati separati dal territorio, grazie ad una legge di riforma sanitaria che ha creato le aziende ospedaliere, e ha creato tutti i presupposti specie economici per una netta separazione dell'assistenza dell'acuto da quella del cronico impedendo di fatto la continuità assistenziale. Questa è una mia analisi personale, credo condivisa da molti, e tutti dovremmo essere consapevoli che questa forma di organizzazione è di per sé un ostacolo molto difficile da superare, perché quando si vuole andare nella pratica, come per esempio sta succedendo nella mia regione coinvolgendo insieme gli ospedali dell'azienda territoriale, le aziende ospedaliere e i medici di medicina generale per costruire $\mathrm{i}$ famosi percorsi diagnostico-terapeutici, emergono sempre fondamentalmente due criticità: una è rappresentata dai cardiologi del territorio, perché i cosiddetti "sumaisti" sono obiettivamente svincolati dal resto del sistema, e sono sostanzialmente deresponsabilizzati nei loro percorsi formativi (da una parte le aziende sanitarie non vogliono investire in formazione in questi professionisti perché li considerano esterni al sistema, dall'altro i sumaisti rivendicano una totale autonomia garantita da uno specifico contratto di lavoro); quindi credo che sia una criticità il non controllo della cardiologia territoriale, quando è così organizzata. Gli altri grandi assenti sono le aziende ospedaliere, perché quando vengono delineati i percorsi diagnostico-terapeutici va ovviamente tenuto conto della loro offerta. Ma questo è oggettivamente molto difficile, essendo gli interessi delle aziende rivolti non tanto ad attività ambulatoriali, a bassa resa economica, ma a tecnologie sempre più mirabolanti e, soprattutto, economicamente redditizie. Il risultato è che tutto il sistema trama per la discontinuità.

Come ultima cosa, vorrei sottolineare una criticità della attuale organizzazione della Cardiologia Riabilitativa nel nostro Paese: la mancanza di flessibilità, argomento di cui ha già accennato Francesco Fattirolli. A questo proposito noi cardiologi riabilitatori dobbiamo convincerci di questa criticità, e devo dire che l'esperienza della creazione di queste linee guida in agenzia è stata per me e molti degli addetti ai lavori un'esperienza illuminante, nel senso che gli epidemiologi clinici dell'agenzia e dell'Istituto Superiore di Sanità, sottolineavano continuamente questo concetto. Dobbiamo dare una risposta in cardiologia riabilitativa: dalla massima complessità alla massima semplicità in termini organizzativi, in modo da dare una risposta adeguata ai bisogni dei pazienti: la cardiologia riabilitativa offre un menu nel quale vanno selezionate modalità di intervento diverse per pazienti che hanno problemi diversi, da quello che abita in cima ad una montagna, a $50 \mathrm{~km}$ dalla cardiologia più vicina, a quello che abita invece in un'area metropolitana iperattrezzata. Credo che noi cardiologi riabilitatori abbiamo poco utilizzato il modello ambulatoriale che è assolutamente vincente trattandosi di un intervento riabilitativo semplice, flessibile, a basso costo, e tremendamente efficace.

\section{BOLOGNESE (GISE)}

Penso che dobbiamo approfittare di questa rara opportunità di vedere attorno ad un tavolo diversi esponenti della comunità cardiologica nazionale, dei medici di medicina generale, per fare quello che gli anglosassoni chiamano dell'outing, e quindi consentitemi di dare una diversa chiave di lettura di alcune delle barriere che sono state fin qui elencate.

$\mathrm{Ci}$ sono ampie sacche di cardiologi che non credono affatto nell'efficacia della riabilitazione cardiologica, perché molti cardiologi ritengono di avere sufficienti capacità per applicare la prevenzione secondaria già all'interno dell'ospedale, di fare counseling, e ritengono che l'intervento riabilitativo, anche quello físico, indirizzato al trattamento ambulatoriale, non sia efficace in tutti i sub-set di pazienti. Questo scetticismo è in parte anche legato al fatto che la riabilitazione cardiologica non ha $\mathrm{i}$ grandi trials che hanno altri componenti della cardiologia. Non è un caso che il trial che stiamo aspettando tutti con grande attenzione sia l'action $H F$, e che si occupi in particolare dello scompenso. Se guardiamo soltanto l'infarto, l'efficacia della riabilitazione cardiologica si poggia su alcuni trials estremamente datati, alcuni in epoca pre-riperfusiva, e con ancora non disponibili tutti gli interventi di ordine farmacologico che oggi sono possibili. Il secondo punto è legato al fatto che esiste un paradosso tra l'importanza che nei Piani Sanitari Regionali Nazionali viene data alla prevenzione secondaria, e il ruolo della riabilitazione cardiologica. La prevenzione secondaria è una priorità assoluta a livello nazionale e regionale però, paradossalmente, la riabilitazione cardiologica ha ancora un ruolo debole nei confronti della comunità cardiologica e soprattutto degli enti regolatori, e quindi dobbiamo spiegarci perché, e in questo la Federazione Italiana di Cardiologia dovrebbe avere un ruolo nel rivitalizzare il ruolo della riabilitazione cardiologica. C'è, infine, il problema delle risorse. Le risorse non sono soltanto scarse, ma quelle esistenti sono disomogenee sul piano nazionale perché riflettono una situazione della riabilitazione cardiologica completamente diversa territorialmente. Si passa da una situazione tipo Veruno, ad una situazione in cui non c'è neanche un ambulatorio dove poter fare un programma ambulatoriale e, quindi, il concetto di flessibilità va anche indirizzato al fatto che andiamo da centri di eccellenza al vuoto sostanziale. In questo senso, mi fa molto piacere quello che ha detto sia Fattirolli che Griffo, perché ho avuto il piacere e il divertimento di partecipare al processo di revisione delle linee guida della riabilitazione. Devo confessare che non ho visto l'ultimo documento definitivo ma una bozza estremamente avanzata. Ebbene, ca- 
so unico nella medicina, un intervento terapeutico come la riabilitazione cardiologica ha la classe di raccomandazione $1 \mathrm{~A}$ in tutti i gruppi di pazienti. Per me è stato veramente elemento di grande meraviglia. Il paziente stabile, con funzione ventricolare sinistra normale, che ha avuto un piccolo infarto miocardico ha un'indicazione $1 \mathrm{~A}$ a fare un intervento di riabilitazione, così come il paziente con grave scompenso, anziano etc. Penso che la soluzione stia nell'identificare delle priorità e su queste investire, cioè dobbiamo identificare quali pazienti abbiano maggiore bisogno di riabilitazione. Attraverso questo, noi riusciremo probabilmente ad estendere il processo di riabilitazione anche se ad altri settori di pazienti. In questo senso io penso che assolutamente la priorità maggiore debba essere ricercata nello scompenso cardiaco.

\section{A. FILIPPI (SIMG)}

Mi sembra che siano stati già espressi chiaramente quali sono i problemi, così come gli obiettivi che tutti noi vogliamo raggiungere. Io mi fermerei soltanto su alcune cose importanti che sono state dette. Il primo punto riguarda le risorse. È stato detto che sono possibili interventi a isorisorse, o con risorse molto scarse, e che questi possono essere efficaci; credo che questo dovrebbe essere il primo punto su cui dovremmo focalizzarci, perché queste sono cose che si possono effettivamente fare. Un'altra cosa molto importante, come ha detto Carlo Schweiger, è che ci sono degli interventi molto semplici che sono estremamente efficaci e largamente disattesi: anche questo è un punto prioritario ed è legato anche alla possibilità di ottenere la disponibilità di risorse, perché credo che saremmo molto più credibili se chiedessimo alla parte pubblica ulteriori investimenti, quando dimostriamo di fare le cose che diciamo di fare e che costano poco. Non sono così ingenuo da pensare che questo sia l'elemento fondamentale, perché sappiamo tutti che le risorse vengono allocate secondo una serie di principi che non sempre rispondono a criteri scientifici. Però, credo che questo sarebbe, da parte della professione, un atto di serietà e di credibilità. Un altro aspetto importante è quello della divisione: lavorare a scatole chiuse è un dato di fatto estremamente rilevante e negativo. Si parlava prima delle nuove linee guida dell'Agenzia Regionale: si tratta di iniziative importanti, ma rivolte soprattutto all'ospedale, con scarso coinvolgimento del territorio. Un altro aspetto molto importante è il fatto che esistono leggi, e documenti, anche molto buoni, che però non funzionano. Partendo da questa situazione, mi sembra che, se vogliamo fare un passo in avanti, dobbiamo fare delle scelte che tengano conto di questi punti. Per quanto riguarda la proposta del Prof. Gensini, cioè di partire utilizzando le risorse che sono presenti in questa sala, le competenze che ci sono, le rappresentanze che ci sono, mi sembra che sia la soluzione ideale. Abbiamo la possibilità di costituire un gruppo di lavoro, che non produce un altro documento, ma che costruisce un percorso di tipo formativo e collaborativo che potrà essere autorevolmente proposto a più livelli: Regione, ASL, realtà locali. E una soluzione che richiede pochissime risorse, ma che può essere il punto di partenza per risolvere i problemi di comunicazione e per proporre modelli di collaborazione e di organizzazione. Questo per fare realizzare quantodetto prima: momenti di incontro tra tutte le persone che sono coinvolte nel programma di riabilitazione, in cui si affrontano non soltanto problemi di tipo culturale (la formazione), perché ormai da questo punto di vista non c'è moltissimo da dire, ma dove si affrontano i problemi concreti, come la flessibilità del modello assistenziale, chi fa che cosa, quale paziente merita una certo intervento, come comunicare, ecc. Queste iniziative possono essere realizzate in brevissimo tempo e possono poi essere la base sia per poter dimostrare di avere fatto già qualche cosa, sia per partire da un'esperienza concreta in cui la realtà viene migliorata, sia per poter premere e ottenere quelle risorse per gli interventi strutturali che poi effettivamente ci servono, Io credo che si possa partire da qui; il prof. Gensini ha un'esperienza immensa da questo punto di vista ed è abilissimo nel riuscire a mettere insieme le componenti necessarie per un progetto di questo tipo. Io credo di potere avere l'appoggio della mia società per questo tipo d'iniziativa.

\section{RICCIO (Area Prevenzione - ANMCO)}

Credo che effettivamente dobbiamo spingere nel diffondere il messaggio culturale che oggi l'intervento strutturato di prevenzione secondaria più efficace è sicuramente un intervento che si svolge nei centri di riabilitazione cardiologica. Lì dove viene effettuato un intervento adeguato di prevenzione secondaria che si basa effettivamente sulla stratificazione prognostica, sull'ottimizzazione della terapia, e sull'adeguato supporto psicologico, io credo che tutto sommato noi possiamo ritenerci soddisfatti che in quel centro è stata fatta un'adeguata prevenzione secondaria. Ora sarebbe sicuramente auspicabile, e credo che sarebbe un enorme successo per la cardiologia riabilitativa, se questa filosofia, se questo tipo di approccio fosse recepito anche nei centri dove la riabilitazione cardiologica non è presente. A me farebbe piacere vedere che $\mathrm{i}$ pazienti non tornino in ospedale dopo un impianto di ICD, perché il defibrillatore ha scaricato appropriatamente, perché, purtroppo, i pazienti non stati preparati in maniera adeguata al fatto che il defibrillatore scarichi, oppure che ritornino in ospedale perché purtroppo nella lettera di dimissione non abbiamo specificato bene, come ha già detto il dott. Filippi, i termini della prescrizione dei farmaci, e soprattutto perché le nostre lettere di dimissione sono assolutamente inadeguate. Tra le cose che i medici di medicina generale oggi ci hanno sottolineato come punto critico e di carenza è sicuramente la stratificazione del rischio; abbiamo mortificato e vanificato lo strumento della Carta del Rischio e Prevenzione, studio italiano che di fatto non è presente nelle lettere di dimissione dei pazienti delle cardiologie italiane. Probabilmente, adeguare il $d a-$ tabase con quelle che sono oggi le evidenze di rivascolarizzazione, o di quanto ci viene dai nuovi farmaci, sarebbe assolutamente auspicabile. L'ulti- 
mo punto riguarda la terza conferenza nazionale sulla prevenzione in materia cardiovascolare; l'Area Prevenzione e il Gruppo italiano di cardiologia riabilitativa hanno messo a punto un percorso per il paziente ad alto rischio. Credo che questo sia uno scenario assolutamente stimolante per la cardiologia, giacché questi pazienti in genere sono mal seguiti, hanno generalmente una sotto-utilizzazione di farmaci e di presidi. Questo percorso andrebbe implementato e diffuso sul territorio, giacché nasce da una conferenza nazionale ed è un documento di consenso tra l'area prevenzione e il GICR. Quindi un impegno dovrebbe essere dedicato anche a questo gruppo di pazienti che ha un profilo di rischio decisamente più alto rispetto al paziente in prevenzione secondaria che già ha avuto un evento, che magari è ben seguito, ben studiato, ben stratificato.

\section{VALFRÈ (Area Chirurgica - ANMCO)}

La legge del consenso informato implica l'obbligatorietà da parte di qualsiasi medico di informare il paziente. L'informazione è alla base della comprensione delle cose anche più difficili, però se non c'è, il paziente resta un pacco postale e non un essere umano, e quindi ecco che mi riallaccio subito alle risorse. 4 anni fa, mi sono posto il problema di come avere una continuità di collegamento col paziente a parità di risorse e ho chiamato il Presidente dell'Ordine dei Medici della mia USL, che non è un'azienda ospedaliera ma è un'azienda territoriale compreso l'ospedale, chiedendo cosa avremmo potuto fare insieme per aiutare e controllare questi pazienti. Mi sono stupito dei dati del collega perché non vi erano il $20 \%$ di irriducibili, il $20 \%$ di volontari, e un $60 \%$ di recuperabili, ma erano $80 \%$ irriducibili, perché tutti mi hanno fatto capire che, senza la retribuzione incentivante, non si collabora. Allora a questo punto abbiamo creato un'attività riabilitativa a livello regionale, per lo meno della nostra realtà dell'USL, e in qualche maniera stiamo rompendo un ghiaccio. Ma tutti noi siamo obbligati nel percorso clinico dell'individuo a dare un messaggio tipo: "Guarda che ti ho fatto qualche cosa, ma non ti ho dato la terapia dell'arteriosclerosi. Il bypass non rappresenta la guarigione dell'arteriosclerosi" e avanti di questo passo, e dire al paziente: "Guarda che ti affido il compito di auto-conservarti, di volerti bene, di aiutarti e di ricorrere periodicamente a qualcuno che ti aiuti a capire". Questo è già un primo passo secondo me; naturalmente il secondo passo è la riformazione di tutti gli specialisti i quali devono spogliarsi della propria autorevolezza o superbia, guadagnare un po' di umiltà e mettersi insieme a condividere un linguaggio che sia trasmissibile al paziente, altrimenti è sterile e continua a rappresentare quel linguaggio dello specialista che si parla addosso di fronte ad altri specialisti. Bisogna veramente parlare in maniera di globalizzazione dell'utilizzo delle risorse. Il Veneto, regione che dal punto di vista della sanità ha sempre viaggiato a vista, ha fatto un qualche cosa di interessante negli ultimi anni, ha fondato l'Osservatorio Epidemiologico. La Società Italiana di Cardiochirurgia ha subito dato il database di tutti i pa- zienti operati nel Veneto all'Osservatorio Epidemiologico. Abbiamo visto come si sono distribuite le spese. Abbiamo realizzato che il 3,6\% dell'intera spesa sanitaria del Veneto viene utilizzata per le malattie cardiovascolari in generale, cioè quasi niente rispetto al fatto che il $49 \%$ della popolazione muore per motivi cardiovascolari. Questa è una consapevolezza che ci ha portato a cercare di proporre qualcosa. Abbiamo così scoperto la prevenzione, mentre i politici non l'hanno scoperta, l'hanno sempre, fin dall'inizio della Costituzione Italiana, enfatizzata. Di fatto, sono state promosse quattro azioni preventive delle malattie infettive trasmissibili e poco o nulla di più; l'igiene pubblica è considerata un qualche cosa di astratto, dove ci sono tanti funzionari e poca attività. La realtà è che questa prevenzione deve far parte delle proprietà individuali di ciascun medico come obbligo ad una professionalità, perché se non la fa è colpevole ed è proprio questo che bisogna cambiare. Bisogna che si porti avanti questo discorso laddove la prevenzione secondaria post-trattamento acuto, qualunque esso sia, deve essere guidata, seguita e deve avere un feed-back. È qui dove la collaborazione tra politica, visione sociale della sanità, e operatori deve trovare un tavolo in comune e legiferare, ma non le leggi scritte bene da qualcuno che è laico, ma leggi che siano fondate sulla praticità di un sistema da sviluppare. Io non ho null'altro da consigliare: finché le risorse sono fisse, ci si dà da fare e si informa soprattutto il paziente, e bisogna prendere atto, colpevolezza o responsabilità a livello delle singole regioni, di come portare avanti quelle risorse della prevenzione che fino ad ora possono essere state utilizzate in maniera fumosa.

\section{MASTROCOLA (Ministero della Salute)}

In relazione alle mie competenze istituzionali tratterò un tema limitato e particolare; tuttavia mi sento coinvolto un po' in tutti gli argomenti discussi nel corso della giornata, quindi farò una brevissima divagazione sulla prevenzione cardiologica, e più in generale sulla prevenzione. È vero quello che diceva prima il professor Valfrè, noi dobbiamo però fare una riflessione. In realtà, il concetto filosofico di prevenzione nelle nostre leggi c'è, a cominciare dalla 833 del '78; infatti, il legislatore all'epoca tra le finalità del Servizio Sanitario Nazionale inserì proprio 1'attività di prevenzione L'art. 32 della Costituzione è abbastanza generico e quindi si preoccupa della tutela dello stato di salute, garantendo, però, cure gratuite alle fasce più deboli. Le leggi di attuazione del dettato Costituzionale, dalla 833/78 in poi, non ultima la 229/1999, fanno della prevenzione l'elemento qualificante del nostro sistema sanitario, la cui preoccupazione è quella di fornire prestazioni di livello in tutti gli interventi di diagnosi, cure, riabilitazione e prevenzione. Per quanto riguarda la prevenzione cardiologica, devo dire che ci sono state e ci sono alcune iniziative dell'Amministrazione Sanitaria Centrale sul tema. Ricordo in proposito, l'iniziativa del collega Donato Greco, Direttore della Prevenzione, e sottolineo che questa nuova concezione della prevenzione è ri- 
scontrabile anche nei fatti e nei provvedimenti adottati dal Ministero; mi piace ricordare che la Direzione Generale dell'Igiene Pubblica è stata soppressa, e si chiama oggi della Prevenzione, quindi anche dal punto di vista ordinamentale il Ministero ha recepito questo principio. Il collega Greco ha attivato un piano per la prevenzione cardiologica, d'intesa con l'Istituto Superiore, grazie ad uno stanziamento dedicato dalla Finanziaria dello scorso anno all'Istituzione del Centro Nazionale per la prevenzione ed il controllo delle malattie. Nell'ambito di questo centro quest'anno vi sono iniziative specifiche sul rischio cardiovascolare; quest'argomento costituisce anche uno dei punti fondamentali del Piano Sanitario Nazionale. L'approvazione del Piano avrà un'altra conseguenza, ovvero l'inserimento dei principi in esso contenuti nel piano nazionale per l'aggiornamento degli operatori sanitari, che è un altro grande impegno da attuare, con conseguente incidenza, sui piani regionali per l'aggiornamento degli operatori sanitari. In tale contesto cambierà pertanto l'ECM e con l'apporto delle regioni sarà possibile introdurre l'accreditamento dei providers, la formazione a distanza e la disciplina degli accrediti. Nel nuovo quadro della formazione il settore della cardiologia dovrà meritare una particolare attenzione ed un posto di rilievo.

Il tema specifico, invece, è quello che i rappresentanti della società di Gruppo Italiano di Cardiologia Riabilitativa e Preventiva hanno posto, prima ai vertici politici del Ministero, e poi a noi tecnici, dirigenti dell'amministrazione, si tratta di un quesito che mi ha dato conoscenza di una problematica che ignoravo. L'istanza è del tutto innocente, per come è stata formulata: "Richiesta di giudizio di merito per la titolarità e responsabilità di governo della riabilitazione cardiologica". In realtà, questa domanda più che un giudizio di merito, richiede l'attivazione di un'iniziativa del Ministero che va ad incidere su un gran numero di interessi. Abbiamo un Consiglio Superiore di Sanità che è deputato a dare questi pareri, quindi il giudizio di merito noi lo abbiamo trasferito a questo organo consultivo. Di fronte a siffatte problematiche il Consiglio investe una sezione che è quella delle professioni. Grazie anche all'iniziativa del Prof. Mario Condorelli, Presidente del CSS, la sezione si è riunita ed ha cominciato l'esame dell'argomento. La parte contro-interessata a questa vicenda ha prospettato le sue tesi, e il giudizio si è fermato perché il Consiglio, con molta prudenza, quando ci sono delle questioni che coinvolgono anche altre categorie, inizia una sorta di audizione, per cui deve acquisire le posizioni sia agli uni che degli altri, e poi integra la propria composizione attraverso esperti che partecipano al procedimento. La questione è, purtroppo, caduta in un momento in cui il Consiglio Superiore ha finito il suo mandato al 31 dicembre, ed è in fase di rinnovo; certamente il governo della riabilitazione cardiologia è problematica da definire, e la sua definizione coinvolge anche l'assetto organizzativo del Servizio Sanitario Nazionale perché, a seconda dell'esito di questo giudizio, in tema di prevenzione cardiologica si dovrà proporre alle regioni un accordo, in quanto per effetto del parere del Consiglio, andrà eventualmente rivisto l'assetto delle strutture del servizio sanitario.
Moderatore: L. TAVAZZI

Richiamo, per comodità di coloro che devono commentare, i punti sollevati che mi sembrano più condivisi o più pregnanti:

1. La storica naturale compartimentalizzazione della cardiologia in vari settori con la difficoltà di comunicazione tra questi, e tra l'insieme della cardiologia ed il mondo esterno.

2. La questione delle risorse, date in misura insufficiente rispetto al peso epidemiologico dell'area cardiovascolare e, usate in modo largamente sproporzionato tra approccio acuto/interventistico e cronico/preventivo.

3. Il problema degli incentivi, che nasce ogni volta che si tenta di introdurre un nuovo raccordo tra area ospedaliera e medici di medicina generale, rischia di paralizzare ogni iniziativa.

4. Il problema dello spazio insufficiente dato alla riabilitazione e alla prevenzione nell'ambito sia della formazione professionale (a livello universitario) che della formazione permanente.

5. Il problema dell'ECM, importantissima iniziativa che in Europa ci ha visto come uno dei primissimi paesi ad applicare questo tipo di continuità della formazione che poi si è inviluppato, prevalentemente per il problema dei provider, e sta rischiando di svuotarsi di contenuto o di venire paralizzato a tempo indefinito.

6. La non condivisione, all'interno dell'area cardiologica, della rilevanza della riabilitazione e del suo ruolo nel contesto della prevenzione. Connesso a questo, la necessità di flessibilizzare e individualizzare i percorsi diagnostico-terapeutici di lungo periodo. E stato toccato il problema del ruolo dei cardiologi extra-ospedalieri che potrebbe essere decisamente rilevante, se fosse integrato, come non è, almeno in alcune aree.

7. Il problema della continuità di informazione con il paziente.

8. Il problema del governo della riabilitazione, che è di fatto il problema chiave per la sopravvivenza stessa della riabilitazione cardiovascolare.

\section{F. CHIARELLA}

Nel primo intervento ho parlato soprattutto di prevenzione per quanto riguarda l'importanza della cultura, del soggetto, delle risorse, mentre qui vorrei andare più all'aspetto riabilitativo in termini interlocutori. Mi è chiaro che cosa si intenda per mentalità, ma meno chiara mi sembra l'identità della riabilitazione, nel senso che abbiamo un problema di riabilitazione ambulatoriale, e un problema di riabilitazione degenziale. Porrei soprattutto la questione su che cosa, dove e quando l'una, e che cosa, dove e quando l'altra. Si tratta di allestire nelle sedi opportune ospedaliere e nei dipartimenti qualche cosa che possa ovunque aiutare il paziente infartuato, ma non ci sono né le risorse, né i centri, né la distribuzione nazionale per fare questo in Italia. Sappiamo che c'è un'allocazione molto asimmetrica di centri di riabilitazione tra nord, centro e sud, e sappiamo invece che il bisogno dei cittadini è ovunque lo stesso, e che dobbiamo ragionare in termini di equità di offerta. È 
anche chiaro quale è il percorso del paziente che ha avuto un intervento cardiochirurgico, mentre meno chiaro quello di chi ha avuto un infarto del miocardio, e meno chiaro ancora è il percorso di chi abbia tutta quella grande problematica di cui si è parlato soprattutto ieri pomeriggio, che è quella dello scompenso. Se dovessi pensare di far accedere il paziente con scompenso cronico, in quelle fasi di scompenso in cui gli ospedali non possono più tenere il paziente perché hanno i loro problemi di servire l'acuto, e dovessero quindi far afferire questo ad un centro di riabilitazione, questo comporterebbe un notevole sconvolgimento nell'architettura delle riabilitazioni in Italia. Quale è quindi l'identità che noi, da questa conferenza, riusciamo a rimettere in discussione sul tavolo e a chiarire tra l'ambulatoriale e la degenziale? e come si possono immaginare i percorsi della degenziale, nella loro completezza in un orizzonte che, in epoca di cardiologia interventistica, il titolo di questa conferenza, è mutato, e rileviamo quanto sia mutato. Quindi diciamo, pongo il problema più che dare risposte.

\section{G. GREGORIO}

Io porrei il problema fondamentale nella ricerca di nuovi modelli organizzativi. Dobbiamo superare modelli organizzativi che consentono lo sviluppo di realtà chiuse, è un discorso che facciamo da sempre. Penso che, quando uno progetta un ospedale in cui vi siano ' $x$ ' posti di chirurgia, si ponga il problema di quante sale operatorie deve fare. Bisognerebbe adottare la stessa logica trasferendola agli atti di programmazione sanitaria. Quando si mette in piedi un percorso per i pazienti cardiovascolari, bisognerebbe pensare a quanti posti per acuti e quanti posti per la riabilitazione, ma bisognerebbe pensare soprattutto agli anelli che tengono insieme il sistema e superare di fatto il modello di dipartimento intramurale. Tutti i dipartimenti, in modo particolare quelli cardiovascolari, dovrebbero raccogliere tutti gli anelli del sistema, superando anche di fatto la lunghezza dello stivale. Pensare ad un modello di dipartimento che raccolga le strutture ospedaliere, le strutture ambulatoriali, analogamente a quanto accaduto per esempio per la salute mentale, pensare a delle strutture intermedie cardiologiche e alla riabilitazione. Che poi questi momenti si integrino in un modo diverso tra di loro è un discorso che verrà col tempo. Ma è fondamentale che vi siano gli strumenti organizzativi per poterlo fare, che vi sia un raccordo per area vasta, per provincia, per azienda, che consenta di fatto agli attori del sistema di mettersi insieme. Tutto il resto viene dopo, perché se noi non cominciamo a parlare tra di noi, e di fatto noi non parliamo tra di noi, i discorsi rimarranno sempre gli stessi. Sono 10 anni, 20 anni che parliamo sempre delle stesse cose sostanzialmente, del fatto dell'importanza della continuità, del fatto che bisogna uscire fuori dall'ospedale; di fatto noi continuiamo a rimanere in ospedale, loro continuano a rimanere negli ambulatori, la riabilitazione continua a rimanere al di fuori. Lo sforzo che bisogna fare è quello di trovare un modello organizzativo che passi attraverso indirizzi nazionali, per la Conferenza Stato-Regioni, ma che obblighi la organizzazione dell'offerta cardiologica in una visione aperta e non più chiusa.

\section{A. BALBARINI}

Credo che il pregio di questo convegno sia stato quello di far emergere delle problematiche importanti, ma secondo me abbiamo anche identificato gli strumenti per poter superare queste problematiche. Queste cose chi le fa? Questo è il punto fondamentale. Vediamo alcuni punti: le risorse sono quelle che sono e non le possiamo cambiare entro certi limiti; la ridistribuzione, quello che ha detto Valfrè è stravolgente, solo il 3\% va al cardiovascolare. Quindi dobbiamo ridistribuire e naturalmente ottimizzare le risorse. I percorsi con il territorio devono essere attuati. Anche questo, chi lo fa? Nella formazione noi iniziamo adesso, non facciamoci delle illusioni. Nella formazione universitaria passerà del tempo, la legge entrerà nel 2007 quindi dovremmo prendere delle iniziative personali. Quello che ha detto Griffo è importantissimo, dobbiamo assicurare una formazione anche ai medici, ai medici che operano, perché Bolognese poi ha detto che ci sono molti medici che non credono nella riabilitazione, e quindi è compito della formazione far sì che questi medici ci credano. Quindi un'altra problematica importantissima è lo scompenso in riabilitazione e, come ha detto Chiarella, questo stravolgerebbe tutti i percorsi perché noi dovremmo "buttare" una quantità di pazienti in strutture che sono totalmente ancora non sufficienti. Quindi, come in molti settori, le problematiche sono individuate, abbiamo individuato anche i sistemi per superare queste problematiche, però poi dobbiamo dire chi le fa queste cose.

\section{A. CAMPANA}

Non aggiungo niente a quanto hanno detto i Colleghi che la riabilitazione la fanno. Mi riallaccio invece a quanto detto da Griffo sulla necessità di modelli differenziati di riabilitazione, non solo per questioni topografiche, come il caso di chi abita a 50 $\mathrm{Km}$ dall'ospedale di riferimento, ma anche per questioni di sub-set di pazienti. I pazienti aritmici gravi, che in genere sono i portatori di ICD e/o di resincronizzatore + ICD, sono dei pazienti scompensati e quindi afferiscono all'area scompenso, che è quella che in genere provvede ad avviare, quando è possibile, la riabilitazione. Tornando ad un altro punto precedentemente affrontato da Carmine Riccio, direi che non è vero che i pazienti non devono tornare al Centro di impianto quando ricevono una scarica dell'ICD (è sbagliato invece se tornano per disinformazione o se tornano al pronto soccorso); devono tornare perché ci deve essere una struttura predisposta come, per esempio, un centro di ascolto, già presente in alcuni ospedali; ciò può determinare nel paziente un grosso miglioramento dal punto di vista psicologico. Un centro di ascolto utilizzabile dal paziente per capire le ragioni di ciò che accade nella interazione tra il suo cuore ed il "device" che gli è stato impiantato; tra l'altro, al di là dell'aspetto psicologico, noi abbiamo il dovere di verificare real- 
mente se la scarica erogata è stata appropriata o meno. Una importanza notevole in questo senso potrebbero averla i futuri sviluppi della telemedicina. In conclusione, è necessario studiare dei modelli differenziati di riabilitazione per i pazienti aritmici che, probabilmente, per quanto ci sembra, devono essere soprattutto dei modelli di riabilitazione che abbiano particolare riguardo per l'aspetto del supporto psicologico.

\section{G. ZITO}

Credo che l'argomento sia stato affrontato e sviscerato da tutti molto bene; si comincia ad intravedere qualche proposta più fattiva per la soluzione del problema. Per quanto riguarda il mio vertice di osservazione, credo che $\mathrm{i}$ punti fondamentali da chiarire siano sostanzialmente tre. Per prima cosa, si è parlato di integrazione tra le varie figure. L'integrazione si avvia quando si dialoga, quando si intravede un soggetto che non fa parte del gruppo e si cerca di intercettarlo, di portarlo attraverso il dialogo al suo interno. I compartimenti stagni e le posizioni un da "barone" sono anacronistiche e non portano da nessuna parte. Ad esempio, sono grato all' ANMCO perché nella consensus sullo scompenso cardiaco ha coinvolto tutte le componenti, dando voce e spazio a tutti. Ci siamo confrontati in modo sereno, in modo tranquillo ed è venuto fuori, a parer mio, un documento di grande spessore. Sono stati intercettati gli attori del discorso, si è ragionato in modo pacato e si è portata avanti un discussione cercando di rispettare le diverse possibilità di intervento ed i ruoli che devono avere le varie componenti. Si è creato un documento che io reputo di grande spessore. Seconda cosa da affrontare è il "problema" della formazione. Non me ne vogliate ma credo che oggi, nella stragrande maggioranza dei casi, in particolar modo per quanto riguarda i corsi ECM, si danno informazioni ma non si fa formazione. Nella realtà si danno una serie di dati a una platea senza badare se la stessa ha recepito o meno il messaggio da portare a casa. Alcune modalità di formazione andrebbero riviste, andrebbero riorganizzate, rifondate. Ripeto, nella stragrande parte di ECM si danno solo sterili informazioni ma non si fa assolutamente formazione. Sotto questo aspetto, sono convinto che le società scientifiche possono svolgere un ruolo importante, lo accennava anche Gensini nel suo intervento. Credo che Tavazzi sia un testimone vivente di quanto è stato complesso il percorso per abbattere alcune barriere ed il Consiglio Federale che è nato nella FIC, rappresenta di certo molto poco ma è anche una grande prospettiva per il futuro. Il ruolo delle società scientifiche va fortemente valorizzato. Credo che queste sono un po' le cose più importanti che sono venute fuori: la formazione, il problema dell'integrazione e il ruolo delle società scientifiche. Le società scientifiche, su questo tema, possono svolgere un ruolo molto importante.

\section{F. FATTIROLLI}

Ritorno su un concetto già espresso e lo puntualizzo. Noi dobbiamo puntare a ottenere un obiettivo concreto che è quello di passare, - come diceva Bolognese poco prima - da una situazione in cui cardiologi sostengono di "non credere" nella riabilitazione, a cardiologi che dicono: "sono a conoscenza che questo paziente, con le sue caratteristiche, con $i$ suoi problemi, otterrà dei vantaggi con la riabilitazione". Sono convinto che la cardiologia riabilitativa ha le capacità di dare un'offerta che riesce ad individuare quelle tipologie di pazienti per i quali questo tipo di assistenza è la migliore offerta possibile. Quindi differenziazione, flessibilità dei programmi, ovviamente un'offerta differenziata anche sulla base della tipologia di pazienti, perché si parla degli ischemici ma anche degli scompensati. È chiaro che questo principio cardine, nel momento in cui viene assunto, determina anche la necessità di dare le risposte. Se noi ci poniamo nell'ipotesi che, faccio ovviamente un paradosso, tutti gli scompensati debbano essere seguiti nell'ambito della cardiologia riabilitativa, facciamo un discorso che è soltanto distruttivo, mentre invece, nel momento in cui cerchiamo di lavorare ad affinare ulteriormente le indicazioni prioritarie, credo che facciamo un'operazione costruttiva. In sintesi, per chiarire questo concetto, la necessità dell'immediato futuro è porre una "gerarchia" di indicazioni alla riabilitazione sulle quali non vi possa essere "opinabilità": mi riferisco quindi ad uno sforzo di elaborazione, che è già in atto, per identificare quelle condizioni cliniche per le quali il percorso riabilitativo è irrinunciabile.

\section{R. GRIFFO}

Sono d'accordo con quanto dice Francesco Fattiroli e direi che uno dei problemi fondamentali è quello che ha tirato fuori Leonardo Bolognese. Questo problema dello scetticismo è un problema reale, però credo che ci siano, come dice Francesco Fattiroli, delle categorie di pazienti sui quali obiettivamente ci siano delle indicazioni indiscutibili e quindi sui quali il percorso va, quanto meno su questi pazienti, applicato. Certo ci sarà tempo per avere nuove informazioni. Lo studio GOSPEL sicuramente ci aiuterà a dare delle risposte con forza e con peso di un grande trial, e quindi qualche informazione sull'efficacia, in tempi moderni, della riabilitazione. Il problema dell'identità del cardiologo riabilitatore, che aveva posto Chiarella, a mio avviso è un problema superato, nel senso che il cardiologo riabilitatore ha ben chiaro quali sono le sue specificità, quale è il suo tipo di intervento, quale è la professionalità e la strutturazione del suo tipo di intervento. Chiarella paventava l'occupazione di tutti i posti di riabilitazione da parte dei pazienti scompensati, ma credo che ci siano anche delle opportunità che vadano anche un po' sfruttate. Non so come sia nelle altre regioni, ma ad esempio da noi c'è un enorme problema, dovranno sparire in Liguria per quest'anno 850 posti letto per acuti, per essere trasformati in posti letto di riabilitazione perché siamo completamente sballati rispetto agli indicatori precisi che pone il piano sanitario. Quindi su questo l'assessore è stato assolutamente categorico, tanto è vero che ci sono reparti ospedalieri di nuova costruzione che erano predestinati a letti per acuti, e si sta già dicendo che 
andranno a fare l'ETD, riabilitazione, cure intermedie, post-acuzie per gli anziani, tutto quello che volete, purché non siano posti per acuti. Ci sono delle opportunità, perché la riabilitazione è una vera emergenza nel territorio per i pazienti, è un'emergenza per il cittadino alla quale bisogna cercare di rispondere. Poi mi tolgo, già che ci siamo tutti, due sassolini dalla scarpa; 1) la Consensus Conference sul documento dello scompenso che è stato appena richiamata. Il nostro gruppo ha saputo dell'esistenza di questo documento, tra cui tra l'altro figura tra $\mathrm{i}$ firmatari di questo documento, non più di una settimana fa. Se vogliamo costruire dei consensi, cominciamo a parlarci con chiarezza tra di noi in modo da sapere esattamente come si partecipa alla elaborazione di un documento. Il secondo riguarda il Piano Nazionale Prevenzione che è stato ricordato più volte. Il Piano Nazionale Prevenzione, che è un argomento che riguarda la prevenzione dell'alto rischio in prevenzione primaria, e riguarda soprattutto un progetto sulla prevenzione secondaria delle recidive, noi del gruppo lo abbiamo scoperto assolutamente per caso, grazie all'attenzione di un nostro associato che girando e navigando in internet ha trovato l'esistenza di questo documento, dandoci il tempo di riuscire ad infilarci nei gruppi regionali che dovevano governare questo tipo di processo che è assolutamente cruciale sul quale noi riabilitatori crediamo di avere delle professionalità assolutamente indiscutibili.

\section{BOLOGNESE}

Credo che si possano dare un paio di messaggi moderatamente ottimisti. Valfrè diceva che in Italia, se non c'è la coercizione, non si riesce a fare nulla, e probabilmente gli enti regolatori, le regioni si stanno rendendo conto di alcuni aspetti che sono legati alla necessità di realizzare una continuità assistenziale. Nella mia regione, ad esempio, uno degli obiettivi prioritari a livello regionale per quest'anno, è la riduzione del tasso di ospedalizzazioni per lo scompenso. Il raggiungimento di questo obiettivo si può realizzare soltanto attraverso la creazione di una continuità assistenziale, di percorsi. Siamo obbligati a farlo, quindi, in questo senso io vedo un certo progredire che dovrebbe portare pian piano a far emergere un ruolo essenziale dei programmi riabilitativi come interventi, in questo caso specifico, capaci di ridurre le ospedalizzazioni. In questo senso sono abbastanza ottimista. Infine il secondo punto che ribadisco, e mi piacciono gli interventi di Francesco Fattiroli e Raffaele Griffo, è la riconversione dei percorsi riabilitativi. I centri di eccellenza di riabilitazione in Italia lo hanno capito. Negli anni ' 80 i centri di eccellenza di cardiologia riabilitativa che io conoscevo ricoveravano tutti i pazienti con infarto trasferiti dall'unità coronarica, o dalla degenza cardiologica, li tenevano lì 10 giorni. Oggi non è più così. Un centro come questo oggi è indirizzato essenzialmente, per esempio, allo scompenso cardiaco. Penso che il ricovero di un soggetto con infarto miocardico acuto, funzione ventricolare sinistra conservata, a cui abbiamo fatto magari una primaria, che è monovaso, a cui si è dato il betabloccante, l'aspiri- na, l'ACE-inibitore, se lo mandiamo a fare la riabilitazione secondo me è uno spreco di risorse. Quindi, vedo come un fatto stranamente positivo la riconversione del percorso riabilitativo, perché ci stiamo già organizzando per questo.

\section{A. FILIPPI}

Sono stato chiamato in causa su un fatto che sento ripetere molto spesso: se si vuol fare qualche cosa con i medici di medicina generale, o li si paga o non si fa niente. Secondo me, si parte in modo sbagliato: se andate a proporre il miglioramento della qualità sulla catena di montaggio alla FIOM CGL, vi chiederanno un aumento. Forse impostare il discorso partendo necessariamente da un tavolo di trattativa aziendale non è sempre la cosa migliore, il che non vuol dire che non debba essere presente il sindacato e non debba essere presente un tavolo aziendale, ma vuol dire che questo approccio è riduttivo e parzialmente fuorviante. Il secondo errore, secondo me, è quello di considerare la medicina generale come un reparto ospedaliero; non lo è. La medicina generale è formata da liberi individui e ciascuno ha la sua impostazione individuale e un suo contesto lavorativo. Quindi, quando si fa una proposta, bisogna tenere in considerazione che questa dovrebbe essere articolata su più piani e che vanno valorizzati gli aspetti che riguardano la professione e l'etica della professione cui nessuno si può sottrarre. In questo senso si può avere una risposta positiva anche ad isorisorse. Nel momento in cui faccio presente ai miei colleghi che il $40 \%$ dei pazienti infartuati non prende l'aspirina, io ho una risposta e questo non mi costa niente. Nel momento in cui però vado a dire che per gestire un paziente devono controllarlo secondo schemi rigidi ogni tre mesi, devono preparare ed iviare un report semestrale, ecc., tutto questo viene percepito come un lavoro aggiuntivo, principalemnte burocratico. In questo caso si richiede un pagamento, non per assistere correttamente i pazienti, ma per adempiere ad una serie di obblighi percepiti come in gran parte estranei ai doveri professionali. Si trattta di due aspetti diversi (etico-professionale ed economico-sindacale) che si devono integrare in modo opportuno. A mio avviso sarebbe necessario trovare gli interlocutori corretti a seconda di quello che si vuole ottenere, identificare aspetti minimi che vanno bene per tutti, e sperimentare aspetti più complessi, sfruttando poi l'effetto "trascinamento". Se tre persone fanno una cosa, nessuno ritiene che sia normale, nel momento in cui diventano $10,20,100$, questo diventa la normalità e rappresenta un nuovo standard di lavoro. Per cui, secondo me, uno dei motivi principali delle difficoltà/fallimenti con i medici di medicina generale è che si si sbagliano sostanzialmente modalità di presentazione e gli obiettivi. Esperimenti per fare le cose in maniera diversa sono molto rari, almeno io non conosco molti, ma quando invece vengono fatti, funzionano e funzionano bene, anche con risorse scarse o nulle. Questo, naturalmente, non vuol dire che non ci debba essere eventualmente anche un incentivo economico, infatti non vedo perché una persona che lavora di più o lavora meglio non debba essere an- 
che pagato di più. Io vengo retribuito sostanzialemnte per quota capitaria, vengo quindi pagato allo stesso modo se lavoro di più o di meno; se lavoro meglio e di più sarebbe una cosa normale e carina ottenere un riconoscimento anche economico. Questo non vuol dire che ogni cosa debba essere necessariamente pagata. $\mathrm{Ci}$ possono essere anche altri aspetti incentivanti, come un ritorno come supporto all'organizzazione/gestione del lavoro e della soddisfazione professionale, che è un incentivo altrettanto importante. In sintesi, a mio avviso, il problema fondamentale è la modalità di approccio sbagliata nei confronti della medicina generale.

\section{RICCIO}

Penso che in questi ultimi anni la cardiologia riabilitativa abbia investito molto in definizioni di percorsi, e in organizzazione, e questo ha dato sicuramente dei grossi risultati. A Maggio all'ANMCO avremo la presentazione dei dati del GOSPEL, credo che sia il momento che il gruppo, che nasce come gruppo di studio, investa di nuovo in ricerca, e quindi invito già da adesso a pensare a qualcosa che possa darci anche delle ulteriori aderenze, poi da presentare a tutta la comunità cardiologica.

\section{VALFRÈ}

Sulla strada del consegnare alla Regione i dati raccolti dalla società, insieme all'Osservatorio Epidemiologico, abbiamo la possibilità di far vedere ai politici e ai governanti come sono andati a finire $\mathrm{i}$ soldi, e la prevenzione rappresenta un modo di investire in risorse, per spendere meno dopo. Per quanto riguarda il rapporto coi colleghi, i colleghi della medicina generale sono uguali a noi, nel senso che sono dipendenti dalla stessa Regione, percepiscono lo stipendio, noi in ospedale lo percepiamo per un risultato che dobbiamo ottenere, se non otteniamo non abbiamo incentivi, e loro dovrebbero avere un budget personale. Il Direttore Generale della nostra ULS, visto che c'era un eccesso di spese farmaceutiche, ha detto ai medici di base: "Se voi sforerete ancora il prossimo anno, vi tagliamo gli incentivi, qualsiasi budget, vi caleremo lo stipendio". L'anno dopo il 30\% dell'abbattimento delle spese farmaceutiche è stato un successo. Aldilà di questo aspetto, dove ovviamente l'orgoglio di appartenenza alla classe medica dovrebbe essere un motivo per lavorare in una certa maniera, credo che la chiave di volta, in questo momento, per sfruttare le risorse esistenti sia che i riabilitatori, che in questo momento stanno lottando per dimostrare la validità della loro multidisciplinarietà, possano funzionare veramente da link con il territorio, il paziente, in modo tale da consentire ai governanti di prendere finalmente delle decisioni in merito a una prevenzione attiva.

\section{MASTROCOLA}

Velocemente 3 punti: governo della Riabilitazione Cardiologica. La questione va posta al Ministro della Sanità, ed al prossimo Consiglio Superiore di Sanità. Da una breve analisi della situazione attuale, consegue che questo quesito va risolto perché c'è una carenza di indicazioni ufficiali e formali di programmi riabilitativo-occupazionali; non se ne parla nel riordinamento delle scuole di specializzazione, operato da una commissione che ha lavorato per cinque anni, presieduta dal Prof. Aldo Pinchera. Non c'è traccia di riabilitazione cardiologica, né a livello di specializzazione, ma, quello che è preoccupante, nemmeno a livello dei servizi, perché per quanto riguarda la cardiologia, tra i servizi equipollenti non figura la cardiologia riabilitativa. È evidente che c'è una lacuna. Non voglio nella discussione che investe le categorie interessate entrare nel merito, questo problema lo affronterà il Consiglio Superiore, certo le istituzioni devono individuare il responsabile nel governo della riabilitazione cardiologica. Credo e dobbiamo batterci perché vi sia un chiarimento sul punto.

Il secondo argomento è quello della formazione. Il futuro prossimo oggi e non domani, deve vedere molto attive le società scientifiche perché, oltre al documento sul riordinamento delle scuole di specializzazione che ha introdotto alcuni concetti fondamentali quali quelli del tronco-comune, la preoccupazione del nostro Ministero e del Consiglio Superiore, è stata quella di evitare che la specializzazione resti sempre a livello molto teorico e didattico e poco pratico. Qualcosa è stato fatto per una formazione sul campo utilizzando le strutture del servizio nazionale, compresi gli IRCCS (Istituti Ricovero e Cura a Carattere Scientifico). Queste iniziative sono apprezzabili ma non sufficienti. Occorre, quindi, porre attenzione ad un procedimento connesso, ossia quello dell'Osservatorio della formazione specialistica, che provvede all'accreditamento delle scuole di specializzazione. Questo è un documento importante perché non può esserci il riordino delle scuole di specializzazione, se non c'è l'accreditamento delle scuole di specializzazione. Si tratta di un provvedimento a livello centrale del MIUR che però coinvolge direttamente gli Osservatori Regionali; perché nella costruzione del legislatore del '99, abbiamo l'Osservatorio Nazionale e gli Osservatori Regionali; a questi ultimi è affidato il compito di controllare se i requisiti delle scuole di specializzazione previsti astrattamente siano poi, in concreto, posseduti. Il futuro deve vedere impegnati tutti, non solo le pubbliche amministrazioni ma anche le società scientifiche a seguire queste problematiche.

Sull'ECM, in estrema sintesi, sono d'accordo con chi ha detto che il Legislatore del '99 ha il merito di aver portato l'educazione continua in medicina in Italia; è stato a mio avviso un Legislatore molto illuminato e molto democratico, forse un po' presuntuoso perché, estendendo l'ECM a tutti gli operatori sanitari, indistintamente, non si è reso conto che forse gestire questo procedimento di circa 850.000 utenti è abbastanza difficoltoso. Tanto vero che la Commissione ECM dell'epoca pensò di sperimentare un sistema incentrato sugli eventi anche se la legge prevedeva che il ruolo fondamentale dell'ECM fosse rappresentato dal provider. Quindi, anche se l'art. 16ter della 229/99 prevedeva l'accreditamento del provider, quella commissione, 
forse preoccupata per la complessità del procedimento decise di sperimentare gli eventi. Dopo cinque anni stiamo ancora sperimentando gli eventi. La sperimentazione è stata molto utile perché ci siamo resi conto di molte incongruenze. Quindi, come dicevo prima, stiamo cercando faticosamente di recuperare, e di risolvere tre problemi: 1) quello dei programmi, perché non si può fare formazione liberamente senza rispettare e delle priorità, che l'amministrazione centrale e le regioni devono dare per quanto riguarda il tema e gli obiettivi della formazione, 2) l'accreditamento dei provider, che significa accreditare un numero ristretto di soggetti, sotto il controllo delle amministrazioni centrali e regionali. Quindi partecipazione attiva dello Stato, delle regioni, e soprattutto degli ordini professionali che avranno il compito di controllare se il provider agisce correttamente nell'accreditamento dell'evento, perché i crediti verranno attribuiti dallo stesso provider, quindi il provider valuta l'evento da lui organizzato e stabilisce quale peso debba avere 3) incentivare la formazione a distanza FAD o sul campo, modalità che economicamente dovrebbero essere più convenienti. Sotto questo profilo, ci sono altri due problemi: quello del costo della formazione, perché nessuno ha stabilito a chi fa carico, e quello del conflitto di interessi che è altra rilevante questione da risolvere. Si tratta di problemi di grande attualità, che rischiano, se non risolti, di incidere negativamente sull'ECM. Insomma questi anni a venire saranno decisivi in sanità, sia per la formazione, sia per la soluzione di alcune problematiche anche di carattere organizzativo del Servizio Sanitario Nazionale, basti pensare alla disponibilità delle risorse economiche.

Concludendo, e tornando al tema principale, la proposta del GICR è stata apprezzata perché non riguarda una rivendicazione di carriera come normalmente fa qualche società scientifica o qualche organizzazione sindacale, bensì manifesta la preoccupazione della categoria di garantire e tutelare la salute del paziente, e credo che questo debba l'intento principale di un operatore sanitario, prescindendo dai risvolti di carriera che pure esistono e sono legittimi. Questo atteggiamento è stato, dunque, apprezzato. Tutti i provvedimenti che dobbiamo fare, devono, comunque, essere oggetto di concertazione, perché nessun provvedimento efficace ed accettabile viene fuori, come dicevano gli antichi, "inascoltata l'altra parte". Purtroppo, devo dirlo, c'è oggi a tutti i livelli una propensione all'assenza di dialogo e questo è un inconveniente che dobbiamo cercare di evitare.

\section{Moderatore: L. TAVAZZI}

Per concludere, toccherò solo pochi aspetti che mi sembrano più rilevanti o più problematici in prospettiva.

Siamo tutti d'accordo sul fatto che i percorsi diagnostico-terapeutici dei cardiopatici disegnano e delimitano le aree di interesse del cardiologo, in sostanza la cosiddetta rete integrata. Io credo che la rete non sia fatta solo da un insieme di operatori, ma da percorsi che passano attraverso unità operative che devono essere collegate in modo operativamente efficace. Non credo che bastino delle buone lettere di dimissione, ma che occorra una rete informatica che consolida i percorsi. Filippi oggi non l'ha detto, ma so che almeno nell'ambito dei loro associati, che sono molti, l'attrezzatura informatica elementare è molto diffusa, l'uso è diventato familiare. Credo che occorra uno sforzo robusto di ulteriore implementazione altrimenti la frammentazione è inevitabile, sta nei fatti. Come diceva Gregorio, sono 20 anni che continuiamo a parlare di integrazione ma non superiamo le compartimentazioni; non lo facciamo per questioni di interesse, di cultura etc, ma anche perché è faticosissimo dal punto di vista operativo. Filippi dice che, quando dimetti un malato, non gli va detto "se hai qualche problema torna da noi", ma va dato il messaggio che il medico di medicina generale è il caregiver, è la persona responsabile e merita questa responsabilità. In realtà, per la grandissima parte dei malati che dimettiamo non abbiamo idea di chi sia il medico di medicina generale a cui il paziente dovrebbe essere affidato e non esiste alcun collegamento con lui. Quindi diventa un esercizio formale quello di delegare responsabilità a chi non si sa chi sia, né se e quanto impegno sia disposto a impiegare per il paziente che stiamo dimettendo. Questo è il primo punto sul quale lavorare, la struttura delle reti integrate.

Il secondo punto riguarda i rimborsi. Se il sistema di rimborso è segmentato in prestazioni, è difficile collegare il tutto. Devo dire che in Lombardia, sta per essere attivata una sperimentazione che riguarda pazienti con scompenso cardiaco (circa 2000) con rimborso a percorsi, definiti temporalmente, della durata di sei mesi, Questo è un primo esperimento che a me pare importante, perché da una struttura economico-organizzativa ai percorsi, riconoscendoli nel loro svolgimento.

Un altro punto nodale è il ruolo dell'Ospedale per il territorio. Nel nostro Ospedale gli ambulatori esterni che si occupano dei bisogni territoriali sono sempre più affollati mentre la ASL si sta sempre più ritirando dall'area cardiovascolare. Quindi è l'Ospedale che sta rispondendo ai bisogni sanitari anche di primo livello, che non richiedono particolare tecnologia o competenze specifiche. I cardiologi extraospedalieri potrebbero svolgere questo compito e in alcune aree, anche se a macchia di leopardo, lo fanno egregiamente.

Il significato e il ruolo della riabilitazione: è un altro punto chiave. Personalmente credo che l'identificazione della riabilitazione con con il trinomio esercizio fisico, dieta e supporto psicologico sia stata nefasta. La riabilitazione cardiologica non è questo. Richiede cultura e professionalità di alto livello ed è ridicolo che si ponga il problema di chi debba gestirla: ovviamente non può che essere il cardiologo. Il fatto che solo si ponga l'ipotesi di un'alternativa fisiatrica, vuol dire non avere compreso la rilevanza e la complessità dei percorsi diagnostico-terapeutici del cardiopatico postacuto e cronico. Ma, se questo problema si pone, parte della responsabilità è dei cardiologi che hanno proposto un'immagine della riabilitazione cardiologica semplificata e sommaria, quindi esercitatile da chiunque. Il problema della riabilitazione è il problema della continuità assi- 
stenziale del cardiopatico cronico. Una riabilitazione cardiologica intesa in senso fisiatrico e gestita da fisiatri costituirebbe una drammatica rottura della continuità assistenziale del cardiopatico. La Federazione Italiana di Cardiologia, che riunisce l'insieme dei cardiologi, deve considerare la riabilitazione una componente vitale della cardiologia e impegnarsi a fondo presso le Commissioni ministeriali e gli Assessorati Regionali alla Sanità perché resti tale.

Per chiudere ottimisticamente, sono d'accordo sul fatto che la sensibilità nei confronti della prevenzione è cresciuta molto negli ultimi anni. Si sono fatte chiacchiere per decenni senza muovere un dito, adesso ho l'impressione che per il convergere di varie istanze: contenere la spesa in prospettiva, soddisfare le aspettative crescenti dei cittadini, disponibilità di dati convincenti sulla praticabilità e sull'efficacia, l'attenzione per la prevenzione: adesso c'è. L'attenzione però deve esprimersi in disponibilità di risorse e questo implica per i cardiologi la necessità di essere presenti e efficaci là dove vengono prese decisioni. Ancora una volta è questione di identità e di rappresentatività. L'efficacia richiede unità di intenti e strutturazione corale delle iniziative. Richiede una Federazione Italiana di Cardiologia unitaria e attiva.

Un ringraziamento particolare và alla Dr.ssa Giuliana Pensa, dell'Agenzia Jean Ann Gilder Scientific Communication Sas - Via Quagliariello 35/E - I-80131 Naples - Italy, per aver curato la trascrizione. 

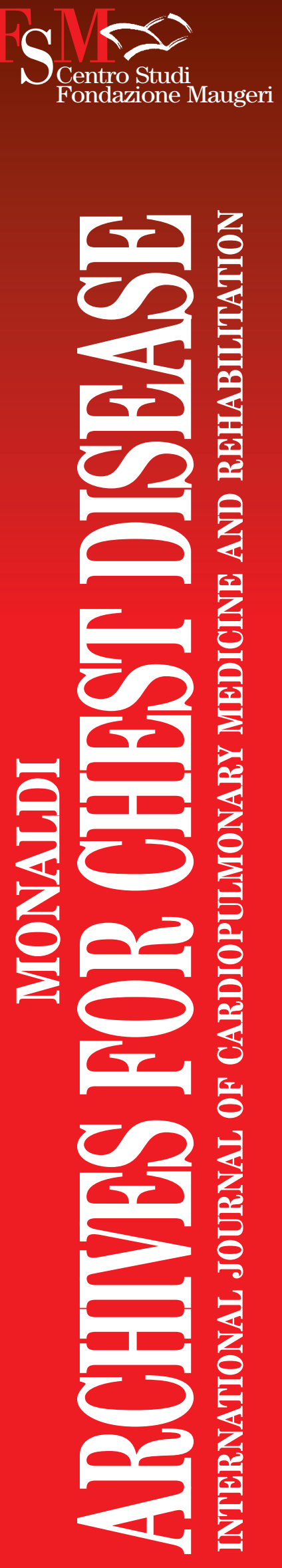

OFFICIAL JOURNAL OF THE ITALIAN WORKING GROUP ON CARDIAC REHABILITATION AND PREVENTION

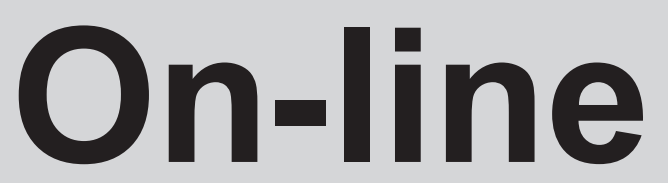

Free abstracts and .pdf full-text articles are available on the web site www.gicr.it

Free registration

The Journal is indexed in: Index Medicus - Medline, Pubmed and Excerpta Medica - Embase [Monaldi Arch Chest Dis] 


\section{Norme per gli Autori}

II Monaldi Archives for Chest Disease è una rivista scientifica pubblicata dal Centro Studi dell'Istituto di Ricovero e Cura a Carattere Scientifico Fondazione Salvatore Maugeri di Pavia e, per quanto attiene alla Sessione Cardiologica, è dal 2002 l'organo ufficiale del Gruppo Italiano di Cardiologia Riabilitativa e Preventiva (GICR).

Pubblica, in lingua italiana e inglese, articoli su argomenti di medicina e di riabilitazione cardiovascolare e polmonare, privilegiando in particolare aspetti di ordine clinico e gestionale.

Gli Archives vogliono rappresentare inoltre un forum per lo scambio di informazioni e conoscenze, anche attinenti alle tematiche di management, di qualità delle cure, aperto a tutte le figure professionali sanitarie che operano nel campo della medicina riabilitativa.

Tutti i contributi dovranno essere preparati secondo le Norme per gli Autori e saranno valutati da peer reviewers, selezionati dagli editors e, nel caso, accettati sulla base di criteri di consenso e di priorità definiti dall'Editorial Board.

I contributi di interesse cardiologico dovranno essere inviati in formato elettronico a:

Editorial Office Monaldi Archives for Chest Disease - Cardiac Series, vigorito@unina.it

I contributi di interesse pneumologico dovranno essere inviati a:

Centro Studi, IRCCS Fondazione Salvatore Maugeri, Via Salvatore Maugeri 4, I-27100 Pavia, Italia, E-mail: monaldi@fsm.it

\section{Sezioni del giornale}

La rivista ospita i seguenti contributi:

Editoriali: articoli o commenti ad invito che rappresentano l'opinione di ricercatori ed esperti riconosciuti.

Articoli originali.

Rassegne: articoli singoli o in serie, organizzati su modello simposio, ad invito o inviati autonomamente o espressione di convegni specifici, in forma di estese revisioni e messe a punto su argomenti di interesse generale nei vari campi della pneumologia e della cardiologia riabilitativa e preventiva.

Opinioni: opinioni di esperti autorevoli in particolari settori che stimolano ancora controversie.

Casi Clinici.

Protocolli: protocolli di studio o ipotesi di lavoro sostenute o suggerite da revisioni critiche della letteratura recente.

Corrispondenza: lettere di commento ad articoli precedentemente pubblicati, ovvero di proposta o di stimolo su temi di interesse generale.

\section{Istruzioni generali}

I contributi a spaziatura doppia con $3 \mathrm{~cm}$ di margine per ogni lato della pagina formato A4, devono essere redatti in formato elettronico (con un word processor) e devono rispondere ai seguenti requisiti generali:

1. Il testo deve essere accompagnato da una lettera indirizzata agli Editors, nella quale l'autore che seguirà la corrispondenza deve indicare la Sezione nella quale l'articolo dovrebbe essere pubblicato e dichiarare che il contenuto del manoscritto è originale, non è stato pubblicato e non è oggetto di valutazione presso altre riviste, eccetto che come abstract.

2. Il testo deve essere redatto nel seguente ordine: a) titolo ed autori nella prima pagina, b) abstract e parole chiave, in inglese e in italiano su fogli separati, c) testo, d) ringraziamenti, e) bibliografia, f) tabelle, g) legende delle figure, h) figure. Le pagine devono essere numerate consecutivamente, partendo da pagina 1 con il titolo.

3. Al momento dell'accettazione dei contributi, tutti gli autori dovranno sottoscrivere a) l'autocertificazione sull'assenza di conflitto di interesse utilizzando il modulo che verrà inviato loro dall'Editorial Office, b) il trasferimento di tutti i diritti di autore alla PI-ME Tipografia Editrice S.r.l. Nessuna parte del materiale potrà essere riprodotta altrove senza il permesso scritto della casa editrice. I suddetti moduli con firma autografa degli autori, devono pervenire in forma cartacea all'Editorial Office.

Allo scopo di mantenere un'adeguata uniformità di stile, l'Editorial Office si riserva di apportare variazioni linguistiche nel testo.

\section{Pagina del titolo}

La pagina del titolo deve comprendere:

1. II titolo.

2. II nome per esteso e cognome degli autori.

3. Un titolo breve di 45 caratteri inclusi gli spazi.

4. I manoscritti in lingua italiana devono riportare il titolo ed il titolo breve anche in lingua inglese.

5. Nome ed indirizzo dell'istituzione presso la quale il lavoro è stato eseguito, con eventuale fonte e natura del sostegno finanziario alla ricerca.

6. Nome, indirizzo postale completo di codice, numero di telefono, di fax ed e-mail dell'autore cui va indirizzata la corrispondenza, indicati a piè di pagina.

\section{Abstract}

1. Editoriali, rassegne, opinioni, casi clinici, e protocolli devono essere accompagnati da un abstract sia in italiano che in inglese, senza abbreviazioni (ad eccezione delle unità standard di misura ed i simboli chimici) e di non più di 300 parole, con inserite, nel rigo finale, da tre a sei parole chiave riportate nell'Index Medicus.

2. Per gli articoli originali, gli abstract devono essere organizzati nel seguente ordine: razionale, materiali e metodi, risultati, conclusioni. Tutti i dati dell'abstract devono essere presentati anche nel testo o nelle tabelle.

\section{Testo}

1. Una lista di abbreviazioni o acronimi quali ECG, LVH, CAD, AMI con la loro definizione deve essere preparata in una pagina a parte.

2. Unità standard di misura e simboli chimici devono essere abbreviati secondo le norme indicate dall'Uniform Requirements for Manuscript Submitted to Biomedical Journal Editors pubblicate in Ann Intern Med 1982; 96: 766-71 e BMJ 1982; 284: 1766-70.

3. I riferimenti bibliografici, le figure e le tabelle devono essere citati nel testo in ordine progressivo, usando numeri arabi per le voci bibliografiche (in apice a testo), per le figure e per le tabelle.

4. II testo dovrà essere strutturato in paragrafi con relativi sottotitoli. II testo di articoli originali dovrà essere suddiviso in sezioni con il seguente ordine: Introduzione, Materiali e Metodi, Risultati e Discussione.

\section{Bibliografia}

1. Le voci bibliografiche devono essere indicate nel testo ed elencate in una sezione separata, in numero progressivo secondo l'ordine di comparsa nel testo.

2. Andranno citati i nomi di tutti gli autori nel caso siano inferiori a sei; nel caso fossero più di sei citare i primi tre ed aggiungere "et al." II titolo deve essere riportato in modo completo.

3. Le abbreviazioni delle riviste devono essere conformi a quelle usate da Index Medicus, National Library of Medicine. Per lo stile e la punteggiatura delle voci bibliografiche seguire gli esempi riportati:

Articoli da riviste

Fornai E, Desideri M, Pistelli F. Smoking reduction in smokers compliant to a smoking cessation trial with nicotine patch. Monaldi Arch Chest Dis 2001; 56: 5-10.

Capitolo da libro

Lown B. Cardiovascular collapse and sudden death. In: Braunwald E, ed. Heart disease. A textbook of cardiovascular medicine. Philadelphia, FA: WB Saunders, 1980: 778-817.

Libro

Pujadas G. Coronary angiography. New York, NY: McGraw-Hill, 1980: 10.

Abstract

Ferrari R, Nayler WG. The protective effect of nifedipine on ischemic and reperfused heart muscle. (abstr) In Abstracts of the International Congress of Pharmacology. Tokio, 1981: 285

\section{Figure}

1. Le figure dovranno essere allegate in formato elettronico compresso (.jpg, .tif, .gif) e il nome del file deve contenere il numero identificativo della figura (es: figura1.jpg)

2. Le figure non devono eccedere la misura di $22 \times 28 \mathrm{~cm}(8.5 \times 11 \mathrm{inch})$. Le lettere devono essere di grandezza sufficiente da permetterne la riduzione. La maggior parte delle illustrazioni saranno ridotte alla larghezza di una colonna.

3. Tutte le linee nei grafici e i disegni devono essere in nero.

4. Le didascalie delle figure devono essere riportate su pagine separate. Tutte le abbreviazioni devono essere riportate e spiegate in ordine alfabetico nella legenda.

\section{Tabelle}

1. Le tabelle devono essere scritte su pagine separate, numerate progressivamente, con titolo allineato a sinistra sulla tabella stessa.

2. Le abbreviazioni devono essere indicate e spiegate in ordine alfabetico sotto alle tabelle, includendo anche la spiegazione dei simboli.

\section{Stampa}

La stampa degli articoli è gratuita. Gli estratti posso essere richiesti alla PI-ME Tipografia Editrice s.r.I., Via Vigentina $136{ }^{\mathrm{A}}$, I-27100 Pavia, Italy; Tel.: +39 0382

572169; Fax: +39 0382 572102; E-mail: tipografia@pime-editrice.it 


\section{Instructions to Authors}

Monaldi Archives for Chest Disease is a scientific journal of the Fondazione Salvatore Maugeri IRCCS, Scientific Institute, Pavia, Italy, and its Cardiac Rehabilitation and Prevention Series is the official journal of the Italian Working Group on Cardiac Rehabilitation and Prevention (G.I.C.R. - Gruppo Italiano di Cardiologia Riabilitativa e preventiva). It publishes in English and Italian original papers on clinical research and health management related to cardiopulmonary medicine and rehabilitation. The journal will also accept well written articles of current opinion, reviews, controversy, educational papers and letters to the editor in pertinent areas of interest. All manuscripts must be written in accordance with the following Instructions to Authors, and will be evaluated by expert Reviewers, selected by the Editors, and eventually accepted on the basis of priority and consensus criteria established by the Reviewers and the Editors.

Cardiology manuscripts should be mailed to the Editorial Office of Monaldi Archives for Chest Disease - Cardiac Series: vigorito@unina.it

Pulmonary manuscripts should be mailed to:

Centro Studi, IRCCS Fondazione Salvatore Maugeri, Via Salvatore Maugeri 4, I-27100 Pavia, Italia, E-mail: monaldi@fsm.it

\section{Journal Sections}

I. Editorials: invited articles or brief editorial comment that represent the opinion of recognized leaders in cardiopulmonary medicine and rehabilitation.

2. Reviews: invited articles by recognized authorities on special topics of general interest. Independent submissions will be considered.

3. Original Articles: original experimental and clinical studies are included in this category.

4. Case Reports: presentation of a clinical case which may suggest novel working hypotheses, with a short discussion of the pertinent literature.

5. Study Protocols: novel study protocols or working hypotheses supported or suggested by recent observations or by a critical review of the literature.

6. Letters to the Editor: these should deal with topics of immediate scientific interest or discuss previously published articles.

\section{General Instructions}

Manuscripts must be typed double-spaced on paper of A4 format, on one side only, with $3 \mathrm{~cm}$ margins on all sides. Manuscripts may be sent by e-mail.

Manuscripts must be accompanied by a covering letter addressed to the Editor, in which the corresponding author must indicate the section in which the paper should be published and state that the paper is not under consideration by any other journal contemporaneously and that it has not been accepted for publication elsewhere, in any language, except as an abstract. The author may suggest the names of Reviewers.

The manuscript must be arranged as follows: a) title page, b) abstract and keywords (in English and Italian language, on separate pages), c) text, d) acknowledgments, e) references, f) tables, g) figure legends, h) figures. Pages should be numbered consecutively, beginning with the title page.

Upon notification of the article's acceptance for publication, all authors will be required to sign a conflict of interest statement (form provided by the Editorial Office) and a copyright declaration transferring all rights to PI-ME Tipografia s.r.I. No part of the published material can be reproduced without written permission from the Publisher. In order to maintain uniformity of style the Editorial Office reserves the right to make, where necessary, linguistic alterations to the manuscript; these will be sent to the authors for approval on revision of proofs.

\section{Title Page}

The title, authors' names (full names) and a short running title less than 45 characters (including spaces) should be typed on the title page. The name and address of the institution from which the work originated, plus information about grants should also be specified. The full name, postal address with zip code, telephone, fax number and e-mail of the author to whom communications, proofs, and requests for reprints should be sent must be typed at the bottom.

\section{Abstract}

Editorials, reviews, opinions, protocols and case reports should be accompanied by an abstract (in English and Italian) of no more than 300 words. Abbreviations (except for standard units of measurement and chemical symbols) should not be used. A list of 3 to 6 keywords should be typed at the end of abstract.

For original articles, a structured abstract should be provided presenting essential data in four paragraphs as follows: Background and Aims, Methods, Results, Conclusions. Complete sentences should be used. All data provided in the abstract must also appear in the manuscript text or tables.

\section{Text}

A list of abbreviations or acronyms such as ECG, LVH, CAD, AMI and their definition must be provided on a separate page. Abbreviations of measurements (e.g. $\mathrm{mm}, \mathrm{kcal}$ ) and chemical symbols should be according to the Uniform Requirements for Manuscripts Submitted to Biomedical Journal Editors published in Ann Intern Med 1982; 96: 766-71 and BMJ 1982; 284: 1766-70.

All references, figures and tables must be cited in the text in numerical order using arabic numerals.

The text of original articles should be subdivided into the following sections: Introduction, Methods, Results and Discussion.

\section{References}

References should be typed double spaced, should begin on a separate sheet and be numbered in the order of appearance in the text. List all authors if 6 or fewer, otherwise list the first 3 and add el al. Abbreviations of journals should conform to those used in Index Medicus, National Library of Medicine. The style and punctuation of references should follow the examples below:

Journal article:

Fornai E, Desideri M, Pistelli F, et al. Smoking reduction in smokers compliant to a smoking cessation trial with nicotine patch. Monaldi Arch Chest Dis 2001; 56: 5-10. Chapter in a Book:

Lown B. Cardiovascular collapse and sudden death. In: Braunwald E, ed. Heart disease. A textbook of cardiovascular medicine. Philadelphia, FA: WB Saunders, 1980: 778-817.

Book:

Pujadas G. Coronary angiography. New York, NY: McGraw-Hill, 1980: 10.

Abstract:

Ferrari R, Nayler WG. The protective effect of nifedipine on ischemic and reperfused heart muscle. (abstr) In Abstracts of the International Congress of Pharmacology. Tokyo, 1981: 285.

\section{Figures}

Figures should be in electronic format (.jpg, .tif, .gif) and the name of the file must contain the figure number (e.g. figure1.jpg). Figures should not be larger than $22 \times 28$ $\mathrm{cm}(8.5 \times 11$ inches). The lettering on the figures should be of sufficient sizes to withstand reduction. Most graphs will be reduced to the width of one column. All line drawings should be in black. Figure title and caption material must appear in the legend, not on the figure, on pages separate from the text. All abbreviations should be explained in the legend in alphabetical order.

\section{Tables}

Tables should be typed on separate pages with the table number and title indicated at the top of the table (aligned to left). Abbreviations should be listed and explained in a footnote in alphabetical order.

\section{Reprints}

Articles will be printed free of charge. Reprints may be ordered from PI-ME Tipografia Editrice s.r.l., Via Vigentina 136', I-27100 Pavia, Italy; Tel.: +39 0382572169 ; Fax: +39 0382 572102; E-mail: tipografia@pime-editrice.it 


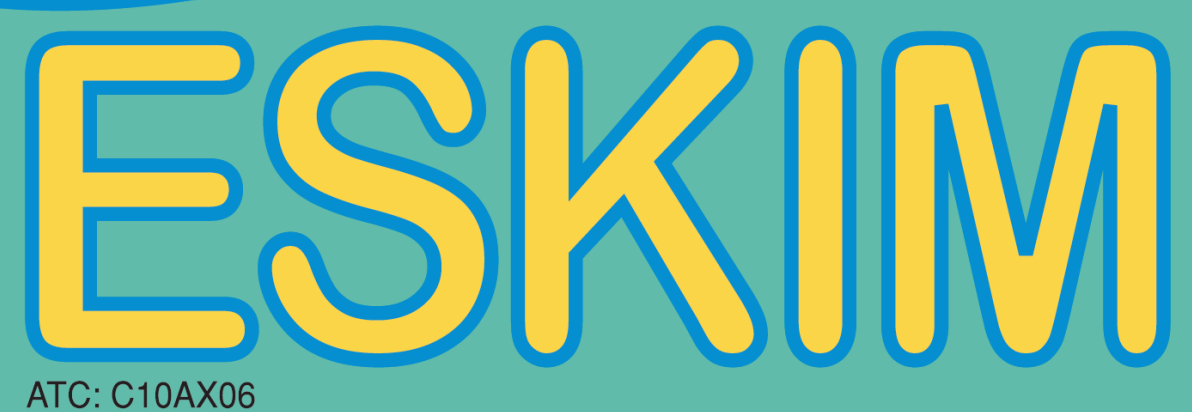

ATC: C10AX06
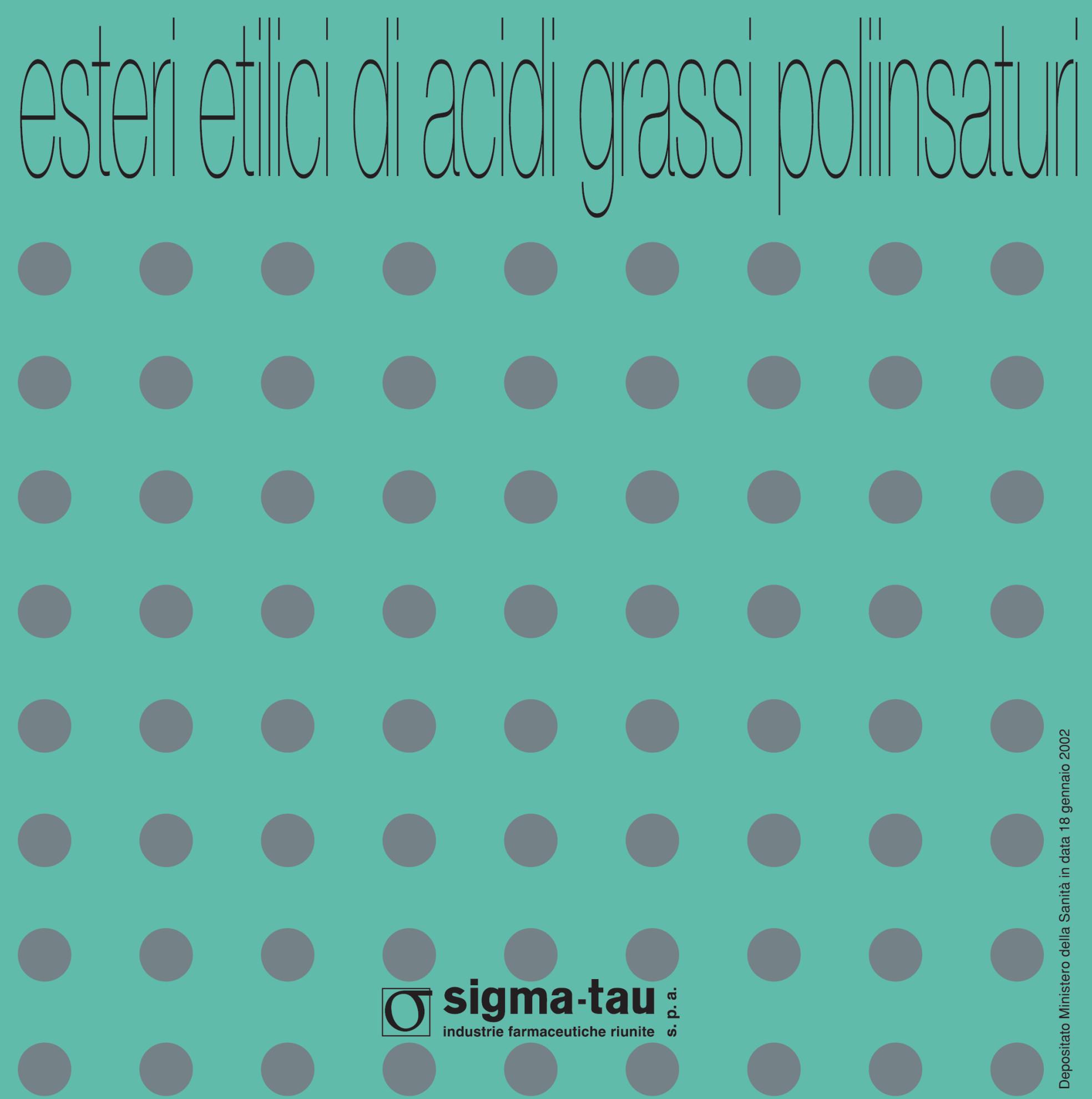

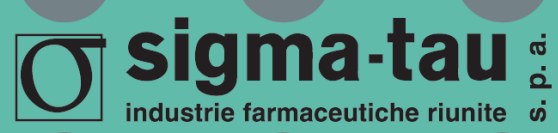



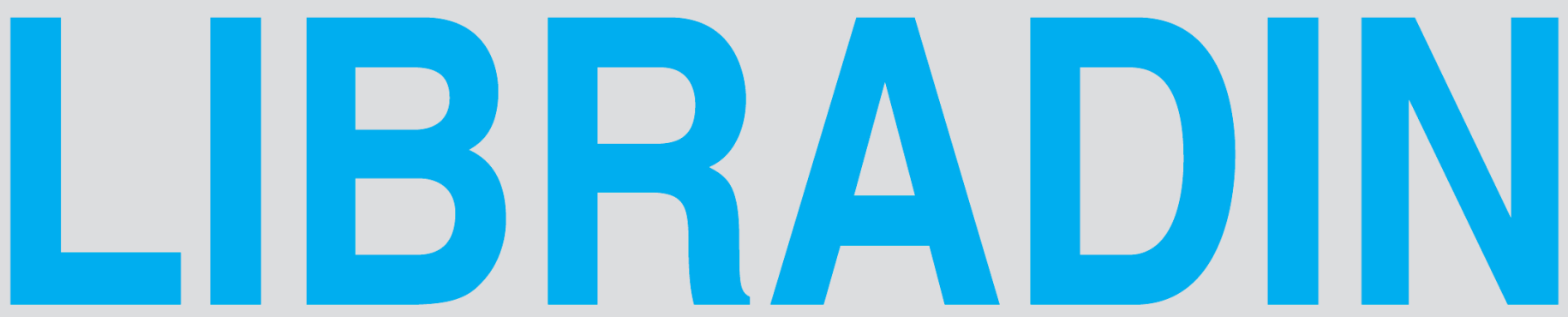

ATC: $\mathrm{C} 08 \mathrm{CA} 12$

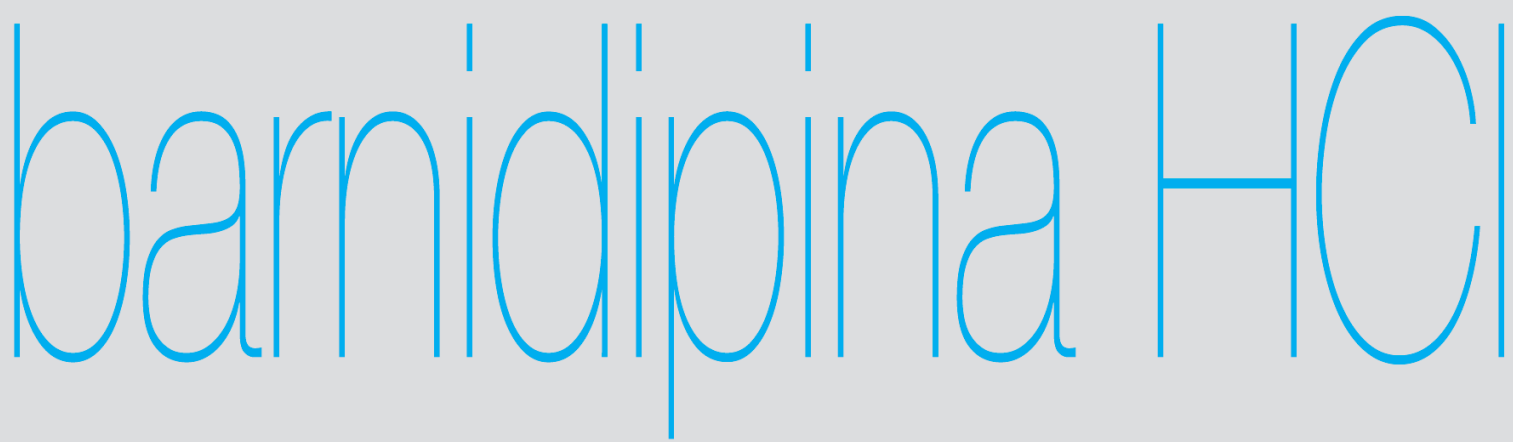




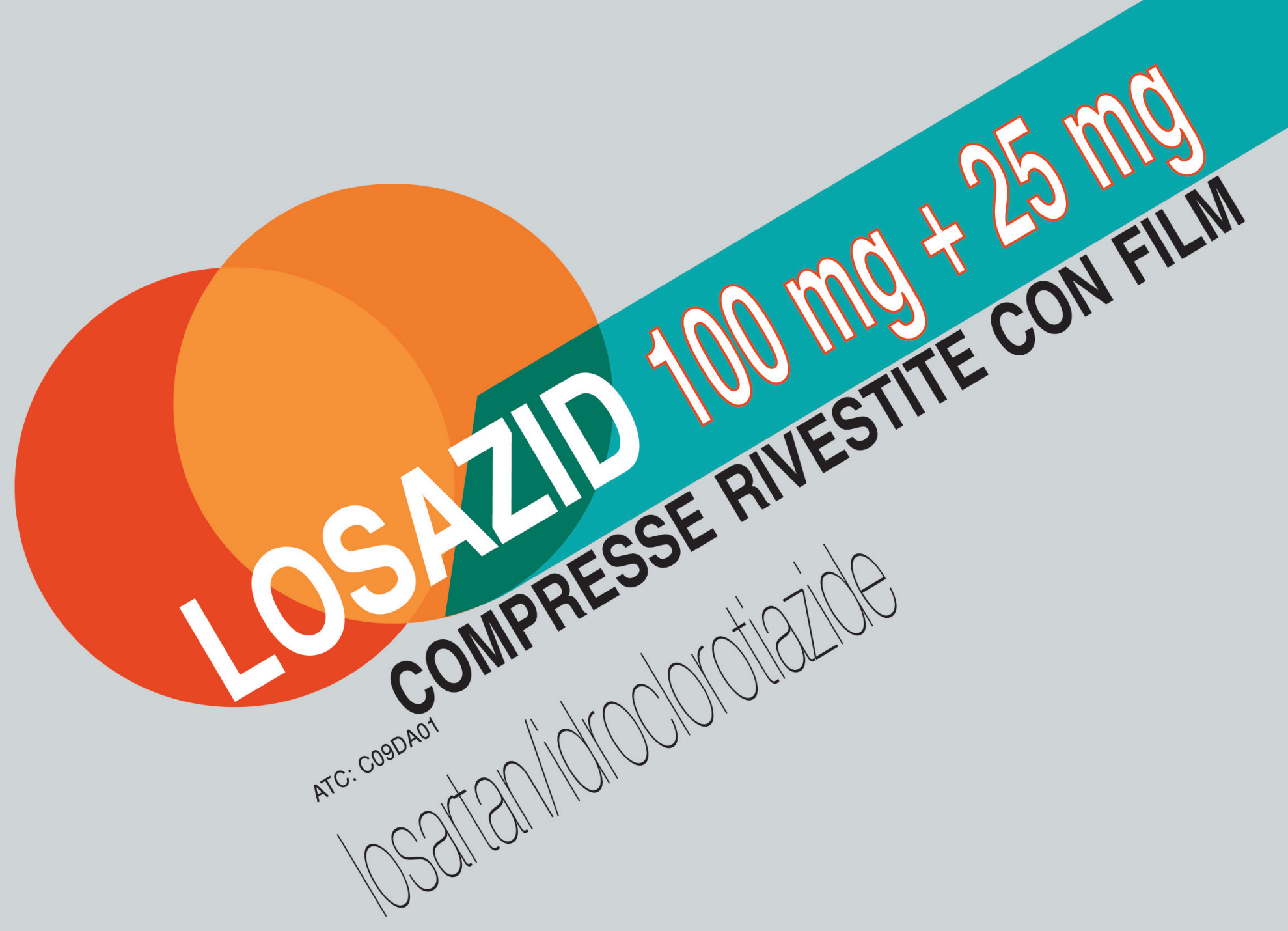

